\title{
BMSAP
}

Bulletins et mémoires de la Société d'Anthropologie de Paris

$16(1-2) \mid 2004$

2004(1-2)

\section{Vestiges humains des niveaux de l'Aurignacien ancien du site de Brassempouy (Landes)}

Human remains from the early Aurignacian levels at the site of Brassempouy

(Landes)

Dominique Henry-Gambier, Bruno Maureille et Randall White

\section{(2) OpenEdition}

\section{Journals}

Édition électronique

URL : https://journals.openedition.org/bmsap/834

DOI : $10.4000 /$ bmsap. 834

ISSN : $1777-5469$

Éditeur

Société d'Anthropologie de Paris

Édition imprimée

Date de publication : 1 juin 2004

Pagination : 49-87

ISSN : 0037-8984

Référence électronique

Dominique Henry-Gambier, Bruno Maureille et Randall White, « Vestiges humains des niveaux de I'Aurignacien ancien du site de Brassempouy (Landes) », Bulletins et mémoires de la Société

d'Anthropologie de Paris [En ligne], 16 (1-2) | 2004, mis en ligne le 13 mai 2008, consulté le 01 juin 2021. URL : http://journals.openedition.org/bmsap/834; DOI : https://doi.org/10.4000/bmsap.834

\section{(c) $(1)$}

Les contenus des Bulletins et mémoires de la Société d'Anthropologie de Paris sont mis à disposition selon les termes de la licence Creative Commons Attribution-NonCommercial-NoDerivatives 4.0 International License. 


\title{
VESTIGES HUMAINS DES NIVEAUX DE L'AURIGNACIEN ANCIEN DU SITE DE BRASSEMPOUY (LANDES) \\ HUMAN REMAINS FROM THE EARLY AURIGNACIAN LEVELS AT THE SITE OF BRASSEMPOUY (LANDES)
}

\author{
Dominique Henry-GAmbIer ${ }^{1}$, Bruno MAUReILle ${ }^{1}$, Randall White ${ }^{2}$
}

RÉSUMÉ

Le gisement de Brassempouy est localisé au sud du département des Landes (France), à deux kilomètres du village de Brassempouy et à quarante kilomètres au sud de Mont-de-Marsan, en Chalosse. Il comprend plusieurs cavités (grotte du Pape, grotte des Hyènes, galerie Dubalen, galerie du Mégacéros) qui appartiennent à un réseau karstique complexe creusé dans une formation calcaire éocène à quelques mètres sous le sol naturel. Les vestiges humains étudiés dans cet article ont été découverts de 1981 à 1996 par H. Delporte dans des niveaux de la grotte des Hyènes, de la galerie Dubalen et de la grotte du Pape attribués à l'Aurignacien ancien. Ces niveaux se placent dans l'intervalle 34000 ans BP-30 000 ans BP.

L'échantillon de vestiges humains comprend 13 dents, un fragment de mandibule, un fragment de crâne et deux phalanges distales de la main représentant des adultes et des enfants. Ces vestiges sont intéressants à un double titre. D'une part, les vestiges humains en contexte aurignacien ancien sont rares et, d'autre part, quatre des dents d'adulte ont une racine perforée ou rainurée intentionnellement et le fragment de crâne présente des cassures effectuées vraisemblablement sur os frais.

L'objectif majeur de cet article est de présenter les caractéristiques morphométriques des différents vestiges et de discuter leur position taxinomique. L'analyse de ces vestiges, en particulier celle des dents isolées, montre qu'ils s'intègrent dans la variabilité des hommes d'anatomie moderne du Paléolithique moyen du Proche-Orient et du Gravettien d'Europe ainsi que dans celle des rares Aurignaciens d'Europe et des Néandertaliens du Würm. Il est impossible de conclure sur leurs affinités taxinomiques. Ailleurs en Europe, les vestiges humains découverts en contexte aurignacien sont aussi très fragmentaires et peu diagnostiques. Les mieux conservés sont en effet souvent mal datés. Aussi l'anatomie des artisans de l'Aurignacien en Europe reste très mal connue de telle sorte qu'il est actuellement impossible d'affirmer que les technocomplexes aurignaciens anciens relèvent uniquement de populations d'anatomie moderne.

Bien que l'analyse détaillée des modifications artificielles soit présentée dans un autre article, on peut d'ores et déjà noter qu'en l'absence de sépultures primaires aurignaciennes, les dents percées de Brassempouy constituent un des rares indices d'intervention anthropique sur le corps, susceptible de relever d'un geste funéraire.

Mots-clés : Anthropologie, Homo sapiens sapiens, Néandertalien, dents, Aurignacien ancien, Gravettien, Moustérien, Châtelperronien, Europe, France, Landes, Brassempouy.

\section{ABSTRACT}

The Brassempouy locality is situated in the Chalosse region in the southern part of the French department of Landes, two kilometers from the village of Brassempouy and forty kilometers south of Mont-de-Marsan. It includes several caves (grotte du Pape, grotte des Hyènes, galerie Dubalen, galerie du Mégacéros) which are part of a complex karstic system

1. UMR 5199 du CNRS, Laboratoire d'Anthropologie des Populations du Passé, Université Bordeaux 1, avenue des Facultés, 33405 Talence CEDEX 01, France, e-mail : d.gambier@anthropologie.u-bordeaux1.fr

2. Department of Anthropology, New York University, 25 Waverly Place, New York, NY 10003, USA. 
developed in an Eocene limestone formation just a few meters beneath the natural ground surface. The human remains described in the present article were discovered by H. Delporte between 1981 and 1996, in levels attributed to the early Aurignacian in the grotte des Hyènes, the galerie Dubalen and the grotte du Pape. These levels date to between 34,000 and 30,000 years $B P$.

The sample of human remains, representing both adults and children, is comprised of 13 teeth, one mandibular fragment, one cranial fragment and two distal phalanges of the hand. The scientific interest of these remains is two-fold. First, human remains are rare in Aurignacian contexts. Second, four of the adult teeth show intentionally perforated or circum-incised roots, and the cranial fragment shows fracture patterns consistant with breakage of fresh bone.

The primary goal of this article is to outline the morphometric characters of the different remains and to evaluate their taxonomic status. The analysis of these remains, especially the isolated teeth, shows that they fall within the range of variation, not only of anatomically modern humans of the Near Eastern Middle Paleolithic and the European Gravettian, but also of the few known European Aurignacians and of the Wurm II Neandertals. It is, therefore, impossible to draw conclusions concerning their precise taxonomic affiliation. Elsewhere in Europe, Aurignacian remains are also very fragmentary and equally undiagnostic. Those that are best preserved are often poorly dated. In sum, the anatomy of the European makers of the Aurignacian is so poorly known that it remains impossible to confirm that the early Aurignacian techno-complex is uniquely the product of anatomically modern populations.

Although the intentional modifications of the Brassempouy human remains are presented in detail in a separate publication, we wish to emphasize here that, with primary Aurignacian burials being completely unknown, the modified human teeth from Brassempouy represent one of the rare traces of human intervention with dead bodies that might be interpreted as funerary behavior.

Keywords: Anthropology, Homo sapiens sapiens, Neandertal, teeth, Early Aurignacian, Gravettian, Mousterian, Châtelperronian, Europe, France, Landes, Brassempouy.

\section{LE GISEMENT}

Il est localisé au sud du département des Landes, à deux kilomètres du village de Brassempouy et à quarante kilomètres au sud de Mont-de-Marsan, en Chalosse, région de l'avant-pays pyrénéen, limitée au nord par l'Adour et au sud par le Gave de Pau (fig. 1). Le gisement s'étend sur environ $2000 \mathrm{~m}^{2}$ sur le versant gauche du vallon du Pouy, affluent du Luy de France, à une cinquantaine de mètres d'altitude. Il comprend plusieurs cavités qui appartiennent à un réseau karstique complexe creusé dans une formation calcaire de l'Éocène à quelques mètres sous le sol naturel.

Du sud au nord-ouest, s'ouvrent la grotte du Pape, la galerie Dubalen, la galerie du Mégacéros et la grotte des Hyènes. Ces cavités communiquent et deux avens ouvrent le réseau sur le versant (fig. 2).

La grotte du Pape, découverte en 1880 à la suite de travaux d'aménagement d'un chemin conduisant à des carrières, débouche par un large porche qui donne accès à la Grande Galerie et à la galerie du Puits. La grotte des Hyènes et la galerie Dubalen, dont les entrées étaient masquées par des colluvions récentes, ont été mises au jour entre 1983 et 1985 (fouilles H. Delporte et D. Buisson). La galerie du Mégacéros découverte en 1997 (fouilles D. Henry-Gambier et F. Bon) est en cours de fouille.

Dubalen, conservateur du Musée de Mont-de-Marsan entreprend les premières fouilles en 1880 (Dubalen 1881). De Laporterie et le $\mathrm{D}^{\mathrm{r}}$ Léon Dufour lui succèdent à partir de 1890 (de Laporterie 1892). En 1892, une excursion des congressistes de l'Association française pour l'Avancement des Sciences, réunie à Pau, se solde par la destruction d'une partie des niveaux archéologiques autour de l'entrée de la grotte du Pape suscitant une controverse parmi les préhistoriens (Delporte 1980, 1996). De 1894 à 1897, Piette et de Laporterie poursuivent des fouilles dans la grotte du Pape, et dans la Galerie « Cro-Magnon », future grotte des Hyènes (Piette, de Laporterie 1895, 1897, 1898 ; Piette 1895, 1896 ; Henry-Gambier, Bon, à paraître). Le site sera ensuite abandonné jusqu'en 1981, année où H. Delporte reprendra des fouilles en collaboration avec D. Buisson. Depuis 1997, les recherches ont lieu sous la direction de D. Henry-Gambier et de F. Bon ${ }^{3}$.

3. Elles associent des chercheurs et des doctorants des Universités de Bordeaux 1, Paris I, Toulouse Paul Sabatier et Toulouse le Mirail, du CNRS, du Laboratoire du Lazaret (Var), du Ministère de la Culture et de l'Université de New York. 


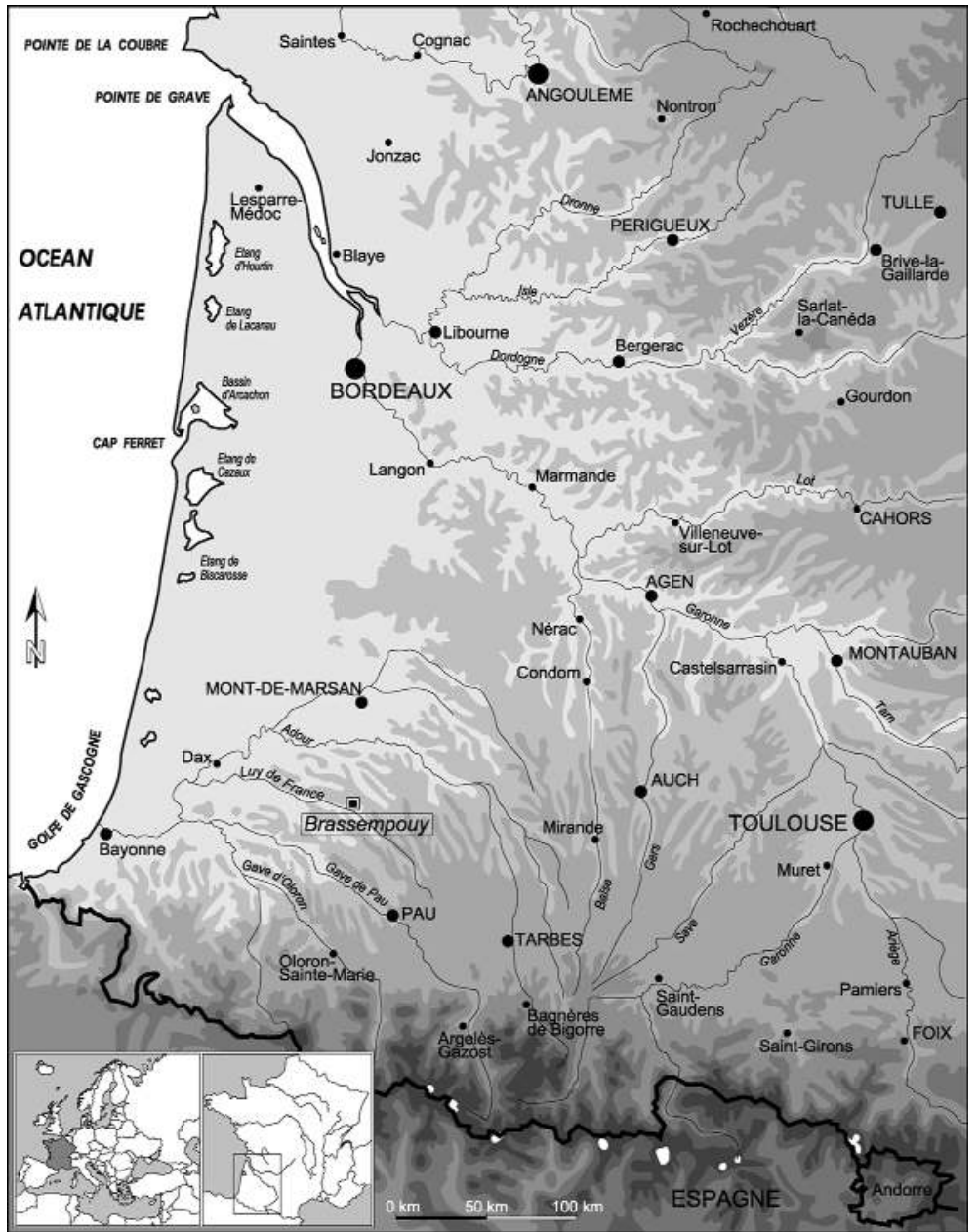

Fig. 1 - Localisation géographique du site de Brassempouy.

Fig. 1-Geographical location of Brassempouy.

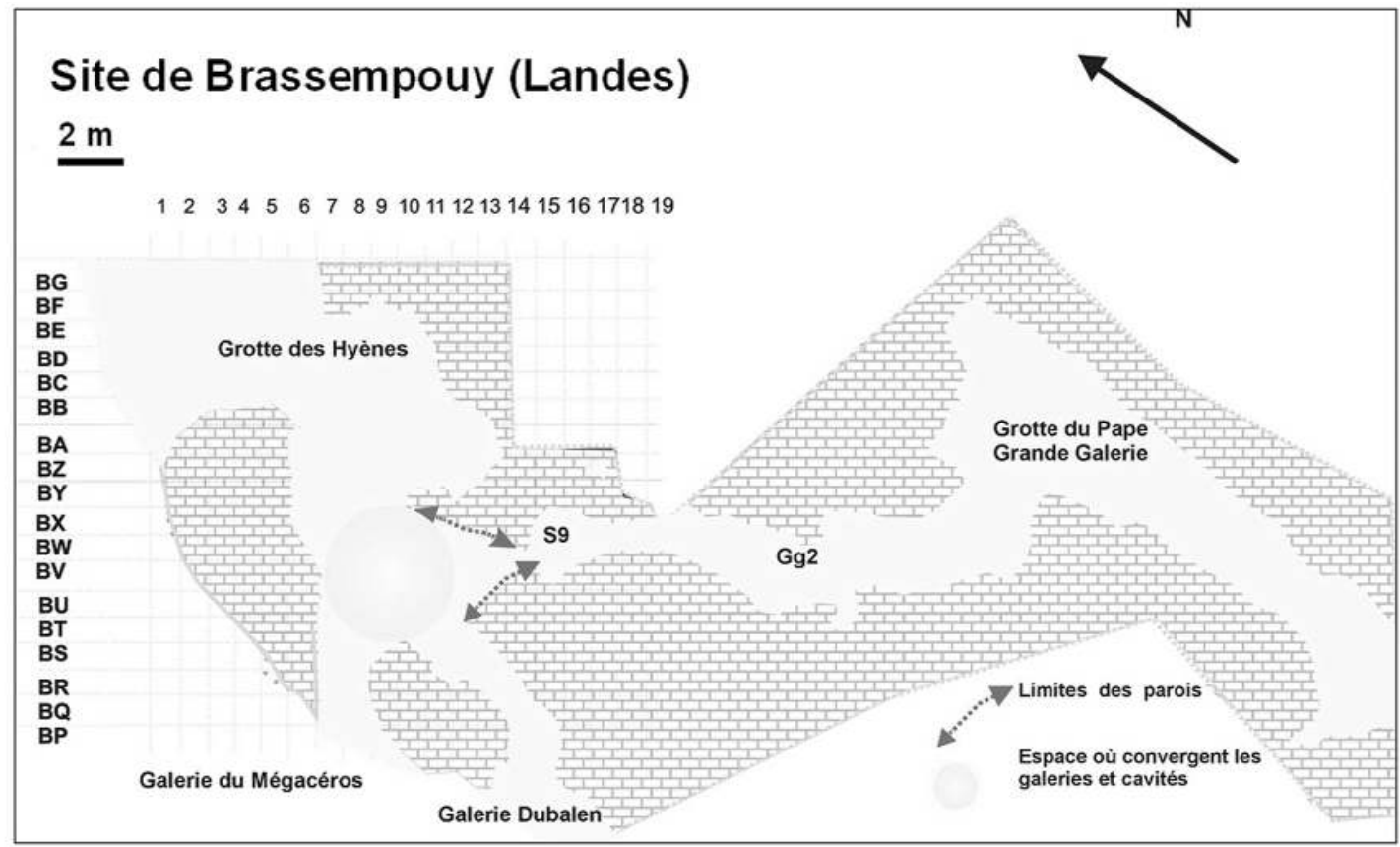

Fig. 2 - Brassempouy, les différentes cavités.

Fig. 2-Brassempouy, the different caves. 
Les publications du $\mathrm{XIX}^{\mathrm{e}}$ s. et l'analyse du matériel des collections Dubalen, de Laporterie et Piette témoignent d'une fréquentation importante du site par les Gravettiens, les Solutréens et les Magdaléniens dans et devant la grotte du Pape. Le Châtelperronien est absent des collections anciennes et l'Aurignacien y est peu représenté (Delporte 1967, 1980, 1987, 1996 ; Merlet 1990). Les fouilles de H. Delporte et de D. Buisson ont confirmé l'importance des occupations gravettiennes et ont démontré que Brassempouy a été occupé dès le début du Paléolithique supérieur. Le Châtelperronien est représenté dans la galerie Dubalen, la Grande Galerie de la grotte du Pape (GG2), la grotte des Hyènes (secteur S8) et dans la galerie du Mégacéros par une série de pointes de Châtelperron (Buisson, Delporte 1990).

L'Aurignacien mis en évidence dans la grotte des Hyènes, dans la galerie Dubalen, dans le secteur GG2 de la Grande Galerie (Buisson 1996) et dans la galerie du Mégacéros est un Aurignacien ancien (Buisson 1996 ; Bon 2002). Aux outils lithiques s'ajoutent des outils en os, en bois de cervidé, de nombreux objets de parure, de l'ocre, des os de faune brûlés ou non qui témoignent d'un large panel d'activités se déroulant sur le site (Bon et al. 1998 ; Bon 2002 ; Henry-Gambier, Bon, à paraître).

Une série de datations C14 (tabl. I) sur os a été réalisée. Les niveaux châtelperroniens se placent dans un intervalle de 31160 à 37510 ans BP (à deux sigmas). Les niveaux aurignaciens de la grotte des Hyènes se situent dans un intervalle de 30000 à 35000 ans BP (à deux sigmas). Deux résultats du niveau $2 \mathrm{E}$ sont aberrants en raison de phénomènes de pollution et/ou de mélange.

Le chevauchement entre les intervalles des dates des niveaux châtelperroniens et aurignaciens indique qu'il est impossible de distinguer chronologiquement l'Aurignacien ancien du Châtelperronien à partir des données radiométriques. Il ne démontre pas, comme cela est souvent écrit, que les niveaux châtelperroniens et aurignaciens sont synchrones. Seules les données stratigraphiques sont de nature à établir la chronologie relative des occupations. À Brassempouy, comme dans tous les sites du Sud-Ouest de la France, les niveaux châtelperroniens sont sous-jacents aux niveaux aurignaciens, excluant une longue contemporanéité entre leurs artisans comme l'a souligné par ailleurs Bordes (2002).

\begin{tabular}{|c|c|c|c|c|}
\hline $\mathbf{N}^{\circ}$ Laboratoire & Niveau & $\begin{array}{l}\text { Âge conventionnel } \\
\text { (BP) }\end{array}$ & sigma & Culture \\
\hline \multicolumn{5}{|l|}{ G. des Hyènes } \\
\hline GIF-9658 & Ens.1 & 30600 & 200 & $\begin{array}{l}\text { Fin des occupations } \\
\text { aurignaciennes }\end{array}$ \\
\hline GIF-8568 & $2 \mathrm{~A}$ & 31820 & 550 & Aurignacien ancien \\
\hline GIF-8174 & $2 \mathrm{~A}$ & 32190 & 620 & Aurignacien ancien \\
\hline GIF-8569 & $2 \mathrm{C}$ & 31690 & 780 & Aurignacien ancien \\
\hline GIF-8570 & $2 \mathrm{E}(* *)$ & 17970 & 150 & Aurignacien ancien \\
\hline GIF/LSM-11035 & $2 \mathrm{E}$ & 31960 & 160 & Aurignacien ancien \\
\hline GIF-9032 & $2 \mathrm{E}(* *)$ & 26870 & 500 & Aurignacien ancien \\
\hline GIF-9031 & $2 \mathrm{D} / 2 \mathrm{E}(*)$ & 30100 & 400 & Aurignacien ancien \\
\hline GifA-98105 & $2 \mathrm{DE}$ & 32410 & 370 & Aurignacien ancien \\
\hline GIF/LSM-11034 & 2DE & 33600 & 240 & Aurignacien ancien \\
\hline \multicolumn{5}{|l|}{ A. Dubalen } \\
\hline GifA-98106 & $\mathrm{I} 2$ & 31520 & 360 & Aurignacien ancien \\
\hline GIF-8171 & $2 b$ & 31990 & 530 & Châtelperronien \\
\hline GIF-9656 & $5 \mathrm{~b}$ & 32680 & 500 & Châtelperronien \\
\hline GifA-101045 & $\mathrm{EBC} 2$ & 36130 & 690 & Châtelperronien \\
\hline
\end{tabular}

Tabl. I - Brassempouy - Datation C14 sur os des niveaux aurignaciens (d'après Fontugne in Henry-Gambier, Bon, à paraître).

Table I-Brassempouy-14C dating of bones from Aurignacian levels (From Fontugne in Henry-Gambier, Bon, in press). 


\section{LES VESTIGES HUMAINS}

L'échantillon comprend des dents isolées ${ }^{4}$ et quatre fragments osseux (tabl. II). Ces vestiges étaient dispersés dans plusieurs carrés et dans plusieurs niveaux.

La majorité d'entre eux proviennent de la grotte des Hyènes, secteur où l'Aurignacien ancien est représenté par une séquence stratigraphique de $1 \mathrm{~m} 50$ d'épaisseur. Les niveaux $2 \mathrm{~A}, 2 \mathrm{C}$ et $2 \mathrm{E}$ (de haut en bas), dont sont issus les restes humains, sont le résultat d'occupations répétées de la grotte par les Aurignaciens.

Une dent isolée et un fragment de mandibule ont été respectivement découverts dans la galerie Dubalen (niveau I1) et au fond de la Grande Galerie de la grotte du Pape (niveau 2F).

Le niveau I1 de la galerie Dubalen correspond au colmatage final de la galerie par des sédiments et des vestiges aurignaciens ayant glissé depuis la grotte des Hyènes, avec laquelle il communique. Le niveau $2 \mathrm{~F}$ du secteur GG2 de la grotte du Pape est formé de sédiment et de matériel archéologique provenant de l'érosion de niveaux d'occupation situés initialement en amont de la galerie (secteur S9) et/ou du versant.

En dépit de leur caractère fragmentaire, ces vestiges sont importants à plusieurs titres :

- ils se placent parmi les plus anciens vestiges humains découverts en Europe occidentale dans un contexte aurignacien ancien bien établi (Henry-Gambier 2002 ; Henry-Gambier, Bon, à paraître) ;

- il est classiquement admis qu'en Europe occidentale, les artisans des techno-complexes de tradition moustérienne (Moustérien tardif, Châtelperronien, Uluzien) sont des Néandertaliens tandis que ceux des premières industries aurignaciennes seraient des hommes d'anatomie moderne. Si le premier terme de cette conclusion s'appuie sur les fossiles de Saint-Césaire en Charente ou Arcy-sur-Cure dans l'Yonne, attribués pour l'instant au Châtelperronien et dont la position taxinomique est solide, il n'en est pas de même pour le second. Les restes humains indiscutablement associés à des niveaux aurignaciens antérieurs à 30000 ans BP sont rares, fragmentaires et/ou rapportables à des immatures. Les spécimens d'anatomie moderne les plus complets (fossiles de Mladeč en République Tchèque ou de Vogelherd en Allemagne) proviennent de fouilles anciennes et leur association à l'Aurignacien reste à démontrer ${ }^{5}$ (Churchill, Smith 2000 ; Henry-Gambier 2002). Les Hommes de Cro-Magnon (France) et de Zlaty Kun (République Tchèque), fossiles d'anatomie moderne assignés à l'Aurignacien ancien, viennent d'être respectivement réattribués au Gravettien et au Magdalénien (Henry-Gambier 2002 ; Svoboda et al. 2002). Ceci doit inciter à la prudence. D'autres spécimens ont été trouvés hors contexte culturel (Hahnöfersand en

\begin{tabular}{|c|c|c|c|c|}
\hline Secteur & $\mathbf{N}^{\circ}$ & Nature & Carré & Couche \\
\hline \multirow[t]{16}{*}{ Grotte des Hyènes } & 884 & M2INFG & BA7 & $2 \mathrm{~A}$ \\
\hline & 69 & DM2INFG & BC9 & $2 \mathrm{~A}$ \\
\hline & 112 & DM1INFG & BD8 & $2 \mathrm{~A}$ \\
\hline & 16 & P2INF D/G & BE4 & $2 \mathrm{~A}$ \\
\hline & 93 & P1/2 SUP D/G & $\mathrm{BZ} 10$ & $2 \mathrm{~A}$ \\
\hline & 344 & Phalange distale main & BE2 & $2 \mathrm{~A} / 2 \mathrm{C}$ \\
\hline & 302 & Phalange distale main & BE4 & $2 \mathrm{~A} / 2 \mathrm{C}$ \\
\hline & 1046 & I1SUPD & BA10 & $2 \mathrm{C}$ \\
\hline & 3040 & P2INFD & BB7 & $2 \mathrm{C}$ \\
\hline & 2879 & DM1SUPG & BB8 & $2 \mathrm{C}$ \\
\hline & 1930 & $\mathrm{M} 1 / 2 / 3 \mathrm{INF} D / \mathrm{G}$ & BB8 & $2 \mathrm{C}$ \\
\hline & 542 & M2SUPD & BF5 & $2 \mathrm{C}$ \\
\hline & 2206 & I1SUPG & BA10 & $2 \mathrm{E}$ \\
\hline & 3625 & DM2SUPG & BA9 & $2 \mathrm{E}$ \\
\hline & 316 & Fragment de voûte crânienne & BY10 & $2 \mathrm{E}$ \\
\hline & 262 & I2SUPG & BY10 & $2 \mathrm{E}$ \\
\hline Grotte du Pape (GG2) & 909 & Fragment de mandibule avec une molaire & S6 & $2 \mathrm{~F}$ \\
\hline Galerie Dubalen & 441 & CSUPG & UW5 & I1 \\
\hline
\end{tabular}

Tabl. II - Brassempouy - Inventaire des vestiges humains par carré et par couche.

Table II-Brassempouy—Inventory of human remains by square and by layer.
4. Bay et Cadenat (1973) signalent une molaire isolée trouvée hors contexte stratigraphique et culturel. Un hémi-maxillaire conservé au Musée des Antiquités Nationales (Collection Piette) proviendrait de la grotte des Hyènes. Son origine stratigraphique est aussi inconnue.
5. Ils pourraient être intrusifs dans des niveaux aurignaciens et donc être plus récents. 
Allemagne ou Cioclovina en Roumanie) et leur ancienneté est très précaire.

Quant aux vestiges fragmentaires représentés en majorité par des dents isolées, ils posent des problèmes non résolus de diagnose taxinomique (Gambier et al. 1990 ; Garralda et al. 1992 ; Gambier 1997, 2000 ; Churchill, Smith 2000 ; Henry-Gambier 2002). Le plus souvent, leur attribution à des populations d'anatomie moderne ne repose pas sur des traits anatomiques mais est déduite de leur association avec l'Aurignacien. Or nous savons d'une part que l'équation «type » humain/technocomplexe lithique n'est pas fondée et que d'autre part dans plusieurs sites, les industries attribuées à une phase très ancienne de l'Aurignacien doivent être révisées et que des problèmes de chronologie existent (Zilhao, d'Errico 1999). En outre, les travaux de technologie lithique en cours ou publiés tendent à montrer que le concept d'unité de l'Aurignacien est aussi à débattre (Bon 2002 ; Bordes 2002). Les relations entre les divers techno-complexes identifiés entre 50000 et 35000 ans en Europe et les relations biologiques entre leurs artisans (Néandertaliens et/ou hommes d'anatomie moderne) s'avèrent en fait beaucoup moins simples que ne le supposaient les travaux plus anciens.

L'identité des Aurignaciens reste donc mal connue. La récente découverte de la mandibule d'Oase 1 (Roumanie) de morphologie moderne (Trinkaus et al. 2003) ne règle pas cette question puisqu'elle a été trouvée hors contexte culturel. Elle atteste cependant de la présence de populations d'anatomie moderne en Europe dès 36 000-32 000 ans BP (à 2 sigmas) mais cet intervalle est compatible aussi bien avec les intervalles de datations connus pour l'Aurignacien ancien qu'avec ceux publiés pour le Moustérien tardif ou les techno-complexes de transition (Zilhao, d'Errico 1999).

- Quatre des dents de Brassempouy et un fragment de crâne portent des traces d'action humaine intentionnelle (perforation, grattage et rainurage) qui attestent peut-être d'un traitement original du corps.

\section{PROBLÉMATIQUE ET MÉTHODES D’ÉTUDE}

L'objectif de cette étude est double :

- en premier lieu, il s'agit de présenter les caractéristiques morphologiques et métriques des dents et des vestiges osseux ainsi que les éventuelles pathologies. L'âge au décès a été déterminé en référence à des standards établis sur des populations actuelles (Ubelaker
1978). Les diamètres des couronnes (diamètres buccolingual et mésio-distal) ont été mesurés au $1 / 10^{\mathrm{e}} \mathrm{de} \mathrm{mm}$. Des radiographies des différents vestiges ont été faites. L'usure dentaire a été évaluée selon l'échelle de Brabant et Salhy (1962).

- en second lieu, l'objectif est de situer les Aurignaciens de Brassempouy parmi leurs prédécesseurs moustériens, leurs «contemporains » et leurs successeurs fossiles et actuels, à partir d'une étude morphométrique comparative des dents. En d'autres termes, il s'agit de tenter de définir leur position taxinomique.

L'analyse technologique des dents perforées n'est pas traitée dans cet article car elle a fait l'objet d'une publication spécifique (White, Henry-Gambier, sous presse).

Pour l'analyse comparative, plusieurs groupes de comparaison ont été constitués (tabl. III, IV, V). La liste des fossiles de comparaison est fournie en fin d'article (annexes I, II).

Groupe 1 : il comprend des échantillons de populations historiques ${ }^{6}$ - Spitalfields, population anglaise du XVII ${ }^{\mathrm{e}}$ au XIX ${ }^{\mathrm{e}}$ s. (Molleson, Cox 1993) Poundbury, population romano-anglaise - Coxyde (groupe monacal de Coxyde en Belgique du XI ${ }^{\mathrm{e}}$ au XII ${ }^{\mathrm{e}} \mathrm{s}$. (Twiesselman, Brabant 1967) ainsi que des individus de populations subactuelles d'Asie du Sud-Est (Bengale) et d'Europe que nous avons pu étudier (base de données P. Semal et B. Maureille).

Groupe 2 : il regroupe les dimensions des dents droites et/ou gauches de 193 populations subactuelles et actuelles de 12 aires géographiques (Kieser 1990). Les paramètres statistiques estimés de ce groupe ont été calculés à partir des effectifs, des moyennes et des écartstypes de chaque population en intégrant les remarques de Cleuvenot et Houet (1993) à propos de la constitution de vastes échantillons, remarques permettant de mieux cerner la variabilité d'une population (base de données B. Maureille).

Groupe 3 : il est constitué par les hommes d'anatomie moderne du Gravettien européen (base de données D. Henry-Gambier, cf annexe I).

Groupe 4 : il comprend les hommes fossiles d'anatomie moderne du Paléolithique moyen du Proche-Orient (base de données B. Maureille, cf annexe I).

6. Données amicalement transmises par P. Semal (IRSNB, Bruxelles, Belgique). 


\begin{tabular}{|c|c|c|c|c|c|c|c|c|}
\hline Fossiles & Variables & $\begin{array}{l}\text { H. actuels } \\
n^{\circ} 1 \\
\text { Effectif }\end{array}$ & $\begin{array}{l}\text { Nombre } \\
\text { Pop. }\end{array}$ & $\begin{array}{l}\text { H. actuels } \\
n^{\circ} 2 \\
\text { Effectif }\end{array}$ & $\begin{array}{l}\text { Nombre } \\
\text { Pop. }\end{array}$ & $\begin{array}{l}\text { H. modernes } \\
\text { (Gravettien) } \\
\text { Effectif }\end{array}$ & $\begin{array}{l}\text { H. modernes } \\
\text { Pal. moy. } \\
\text { Effectif }\end{array}$ & $\begin{array}{c}\text { Néandertaliens } \\
\text { Würm ancien } \\
\text { Effectif }\end{array}$ \\
\hline \multirow{4}{*}{$\begin{array}{l}\text { Abri Dubalen } \\
441 \text { CSUP }\end{array}$} & MD & 322 & 5 & 10466 & 178 & 24 & 9 & 25 \\
\hline & $\mathrm{BL}$ & 326 & 5 & 6397 & 116 & 23 & 9 & 31 \\
\hline & IND & 317 & 5 & & & 21 & 9 & 25 \\
\hline & ROB & 317 & 5 & & & 21 & 9 & 25 \\
\hline \multirow{4}{*}{$\begin{array}{c}\text { Grotte des Hyènes } \\
1046 \text { I1SUP }\end{array}$} & MD & 297 & 5 & 10706 & 174 & 24 & 11 & 30 \\
\hline & $\mathrm{BL}$ & 323 & 5 & 4601 & 91 & 24 & 10 & 33 \\
\hline & IND & 297 & 5 & & & 24 & 10 & 27 \\
\hline & ROB & 297 & 5 & & & 24 & 10 & 27 \\
\hline \multirow{4}{*}{$\begin{array}{l}\text { Grotte des Hyènes } \\
2206 \text { I1SUP }\end{array}$} & $\mathrm{MD}$ & 297 & 5 & 10706 & 174 & 24 & 10 & 30 \\
\hline & $\mathrm{BL}$ & 323 & 5 & 4601 & 91 & 24 & 7 & 33 \\
\hline & IND & 297 & 5 & & & 24 & 7 & 27 \\
\hline & ROB & 297 & 5 & & & 24 & 7 & 27 \\
\hline \multirow{4}{*}{$\begin{array}{c}\text { Grotte des Hyènes } \\
262 \text { I2SUP }\end{array}$} & MD & 302 & 5 & 10590 & 176 & 21 & 10 & 25 \\
\hline & BL & 320 & 5 & 4568 & 95 & 23 & 10 & 29 \\
\hline & IND & 301 & 5 & & & 21 & 10 & 22 \\
\hline & ROB & 301 & 5 & & & 21 & 10 & 22 \\
\hline \multirow{4}{*}{$\begin{array}{l}\text { Grotte des Hyènes } \\
3040 \text { P2INF }\end{array}$} & $\mathrm{MD}$ & 323 & 5 & 10256 & 176 & 23 & 6 & 48 \\
\hline & BL & 320 & 5 & 7923 & 141 & 24 & 6 & 51 \\
\hline & IND & 318 & 5 & & & 24 & 6 & 48 \\
\hline & ROB & 318 & 5 & & & 24 & 6 & 48 \\
\hline \multirow{4}{*}{$\begin{array}{c}\text { Grotte des Hyènes } \\
542 \text { M2SUP }\end{array}$} & $\mathrm{MD}$ & 303 & 5 & 9105 & 160 & 27 & 9 & 33 \\
\hline & $\mathrm{BL}$ & 305 & 5 & 7659 & 141 & 30 & 9 & 32 \\
\hline & IND & 301 & 5 & & & 27 & 9 & 31 \\
\hline & ROB & 301 & 5 & & & 27 & 9 & 31 \\
\hline \multirow{4}{*}{$\begin{array}{l}\text { Grotte des Hyènes } \\
884 \text { M2INF }\end{array}$} & MD & 300 & 5 & 8169 & 162 & 34 & 4 & 55 \\
\hline & $\mathrm{BL}$ & 304 & 5 & 7430 & 140 & 34 & 4 & 55 \\
\hline & IND & 300 & 5 & & & 34 & 4 & 54 \\
\hline & ROB & 300 & 5 & & & 34 & 4 & 54 \\
\hline \multirow{4}{*}{$\begin{array}{c}\text { Grotte des Hyènes } \\
2879 \text { DM1SUP }\end{array}$} & $\mathrm{MD}$ & 100 & 5 & 344 & 10 & 7 & 5 & 22 \\
\hline & $\mathrm{BL}$ & 101 & 5 & 345 & 10 & 7 & 4 & 23 \\
\hline & IND & 96 & 5 & & & 6 & 4 & 22 \\
\hline & ROB & 96 & 5 & & & 6 & 4 & 22 \\
\hline \multirow{4}{*}{$\begin{array}{c}\text { Grotte des Hyènes } \\
3625 \text { DM2SUP }\end{array}$} & MD & 87 & 5 & 351 & 10 & 8 & 5 & 24 \\
\hline & $\mathrm{BL}$ & 88 & 5 & 351 & 10 & 9 & 5 & 24 \\
\hline & IND & 86 & 5 & & & 7 & 5 & 23 \\
\hline & ROB & 86 & 5 & & & 7 & 5 & 23 \\
\hline \multirow{4}{*}{$\begin{array}{c}\text { Grotte des Hyènes } \\
112 \text { DM1INF }\end{array}$} & MD & 107 & 5 & 331 & 10 & 4 & 6 & 21 \\
\hline & $\mathrm{BL}$ & 109 & 5 & 346 & 10 & 4 & 6 & 21 \\
\hline & IND & 107 & 5 & & & 4 & 6 & 21 \\
\hline & ROB & 107 & 5 & & & 4 & 6 & 21 \\
\hline \multirow{4}{*}{$\begin{array}{l}\text { Grotte des Hyènes } \\
69 \text { DM2INF }\end{array}$} & $\mathrm{MD}$ & 102 & 5 & 350 & 10 & 8 & 5 & 22 \\
\hline & $\mathrm{BL}$ & 102 & 5 & 350 & 10 & 7 & 5 & 22 \\
\hline & IND & 101 & 5 & & & 7 & 5 & 21 \\
\hline & ROB & 101 & 5 & & & 7 & 5 & 21 \\
\hline
\end{tabular}

Tabl. III - Effectifs des échantillons de comparaison par type de dents étudié. Nombre Pop. = nombre de populations, $M D=$ diamètre mésio-distal de la couronne, $B L=$ diamètre bucco-lingual de la couronne, $I N D=$ indice de la couronne $[B L / M D \times 100], R O B=$ robustesse de la couronne $=B L \times M D$.

Table III-Numbers of comparative samples for type of teeth studied. Nombre Pop. = number of populations, $M D=$ mesio-distal diameter of the crown, $B L=$ bucco-lingual diameter of the crown, $I N D=$ index of the crown $[B L / M D \times 100], R O B=$ robustness of the crown $=B L \times M D$. 
Groupe 5 : il est formé des fossiles humains de la lignée néandertalienne du Würm (base de données B. Maureille, cf annexe I).

Groupe 6 : il comprend les restes dentaires aurignaciens (base de données D. Henry-Gambier, tabl. $I V, V$ ).

Les bases de données des groupes 3, 4, 5 et 6 résultent de la compilation de données bibliographiques et des observations de deux des auteurs. Les mensurations des dents non publiées par les auteurs sont extraites des articles princeps sur les fossiles.

Le nombre d'individus fossiles étant en général faible, les dents droites et gauches des groupes fossiles ont été considérées séparément. Un individu pourra ainsi être représenté par deux séries de valeurs (dent droite et dent gauche). En revanche, dans le groupe 1 de populations actuelles chaque individu est représenté par la moyenne des valeurs de la dent droite et de la dent gauche. Dans le groupe 2, les dents ne sont pas latéralisées.

Quatre variables ont été retenues : les diamètres bucco-lingual (BL) et mésio-distal (MD) ainsi que la robustesse de la couronne (BL x MD) et l'indice coronaire BL/MD x 100.

Pour chaque paramètre, l'estimation de la position des dents de Brassempouy par rapport aux groupes de référence, excepté pour le groupe 6 (Aurignaciens d'Europe) dont l'effectif est trop faible, a été évaluée à l'aide du test de Student (alpha $=0,05)$, selon un

\begin{tabular}{|c|c|c|c|c|c|c|c|c|c|c|c|c|c|c|}
\hline Site & Individu & Dent & & & BL & MD & IND & ROB & $\begin{array}{l}\text { Nombre } \\
\text { Cuspides }\end{array}$ & $\mathrm{R}$ & Âge dentaire (ca) & Dysplasie & Pays & Références \\
\hline Brassempouy & 2879 & DM1 & SUP & G & 9,9 & $\mathbf{7 , 5}$ & 132,0 & 74,3 & 3 & 3 & 1 an \pm 4 mois & non & France & \\
\hline Brassempouy & 3625 & DM2 & SUP & G & 10,0 & 8,4 & 119,0 & 84,0 & $4 ?$ & lysées & 10 ans \pm 30 mois & non & France & \\
\hline Caminade Ouest & 1 & DM2 & SUP & D & NM & NM & & & $?$ & lysées & 10 ans \pm 30 mois & non & France & Obs. Henry-Gambier \\
\hline Ksar' Aqil & 1 & DM2 & SUP & D & 9,9 & 9,1 & 108,8 & 90,1 & 4 & lysées & 10 ans & non & Liban & Tillier, Tixier 1991 \\
\hline Brassempouy & 112 & DM1 & INF & G & 7,8 & 8,4 & 92,9 & 65,5 & ? & lysées & 10 ans \pm 30 mois & non & France & \\
\hline Isturitz 2000 & 1 & DM1 & INF & $\mathrm{D}$ & 7,1 & 8,1 & 87,7 & 57,5 & $?$ & lysées & 10 ans \pm 30 mois & non & France & Obs. Henry-Gambier \\
\hline Cueva del Castillo & 2 & DM1 & INF & $\mathrm{D}$ & 7,0 & 9,0 & 77,8 & 63,0 & 5 & ? & $4-5$ ans & non & Espagne & Garralda et al. 1992 \\
\hline La Quina & 761 & DM1 & INF & $\mathrm{D}$ & 7,2 & 9,0 & 80,0 & 64,8 & 5 & 2 & $>6$ ans, $<12$ ans & non & France & Obs. Tillier \\
\hline Bacho Kiro & 1124 & DM1 & INF & G & 7,5 & 8,8 & 85,2 & 66,0 & $?$ & $?$ & Immature & ? & Bulgarie & $\begin{array}{l}\text { Glen, Kaczanowski } \\
1982\end{array}$ \\
\hline Brassempouy & 69 & DM2 & INF & G & 9,8 & 10,4 & 94,2 & 101,9 & 4 & lysées & 10 ans \pm 30 mois & non & France & \\
\hline La Quina & 25 & DM2 & INF & G & $\mathrm{NM}$ & NM & & & 4 ou 5 & ? & $>6$ ans, $<12$ ans & non & France & Obs. Henry-Gambier \\
\hline Les Rois & A & DM2 & INF & $\mathrm{D}$ & 9,5 & 10,0 & 95,0 & 95,0 & $5 ?$ & 2 & 10 ans \pm 30 mois & non & France & $\begin{array}{l}\text { Vallois 1958/ } \\
\text { Obs. Henry-Gambier }\end{array}$ \\
\hline Les Rois & A & DM2 & INF & G & NM & NM & & & $5 ?$ & 2 & 10 ans \pm 30 mois & non & France & $\begin{array}{l}\text { Vallois 1958/ } \\
\text { Obs. Henry-Gambier }\end{array}$ \\
\hline Les Rois & $\mathrm{R} 50 / 33$ & DM2 & INF & G & 10,0 & 11,5 & 87,0 & 115,0 & 5 & 2 & 8 ans \pm 30 mois & non & France & Obs. Henry-Gambier \\
\hline Fontéchevade & 2 & DM2 & INF & $\mathrm{D}$ & 9,1 & 10,4 & 87,5 & 94,6 & 5 & 2 & 4 ans \pm 12 mois & non & France & Obs. Henry-Gambier \\
\hline Cueva del Castillo & 2 & DM2 & INF & $\mathrm{D}$ & 9,0 & 11,0 & 81,8 & 99,0 & 4 ou 5 & $?$ & $4-5$ ans & non & Espagne & Garralda et al. 1992 \\
\hline La Quina & 761 & DM2 & INF & $\mathrm{D}$ & 9,5 & 10,5 & 90,5 & 99,8 & 5 & 2 & $>6$ ans, $<12$ ans & non & France & Obs. Tillier \\
\hline Bacho Kiro & 559 & DM2 & INF & $\mathrm{D}$ & 9,5 & 10,0 & 95,0 & 95,0 & $?$ & $?$ & Immature & $?$ & Bulgarie & $\begin{array}{l}\text { Glen, Kaczanowski } \\
1982\end{array}$ \\
\hline
\end{tabular}

Tabl. IV - Europe, caractéristiques des dents déciduales aurignaciennes. MD = diamètre mésio-distal de la couronne, $B L=$ diamètre bucco-lingual de la couronne, IND = indice de la couronne [BL/MD $x$ 100], $R O B=$ robustesse de la couronne $=B L \times M D, R=$ nombre de racines.

Table IV-Europe, characteristics of Aurignacian deciduous teeth. MD = mesio-distal diameter of the crown, BL = bucco-lingual diameter of the crown, IND = index of the crown $[B L / M D \times 100], R O B=$ robustness of the crown $=B L \times M D, R=$ number of roots. 


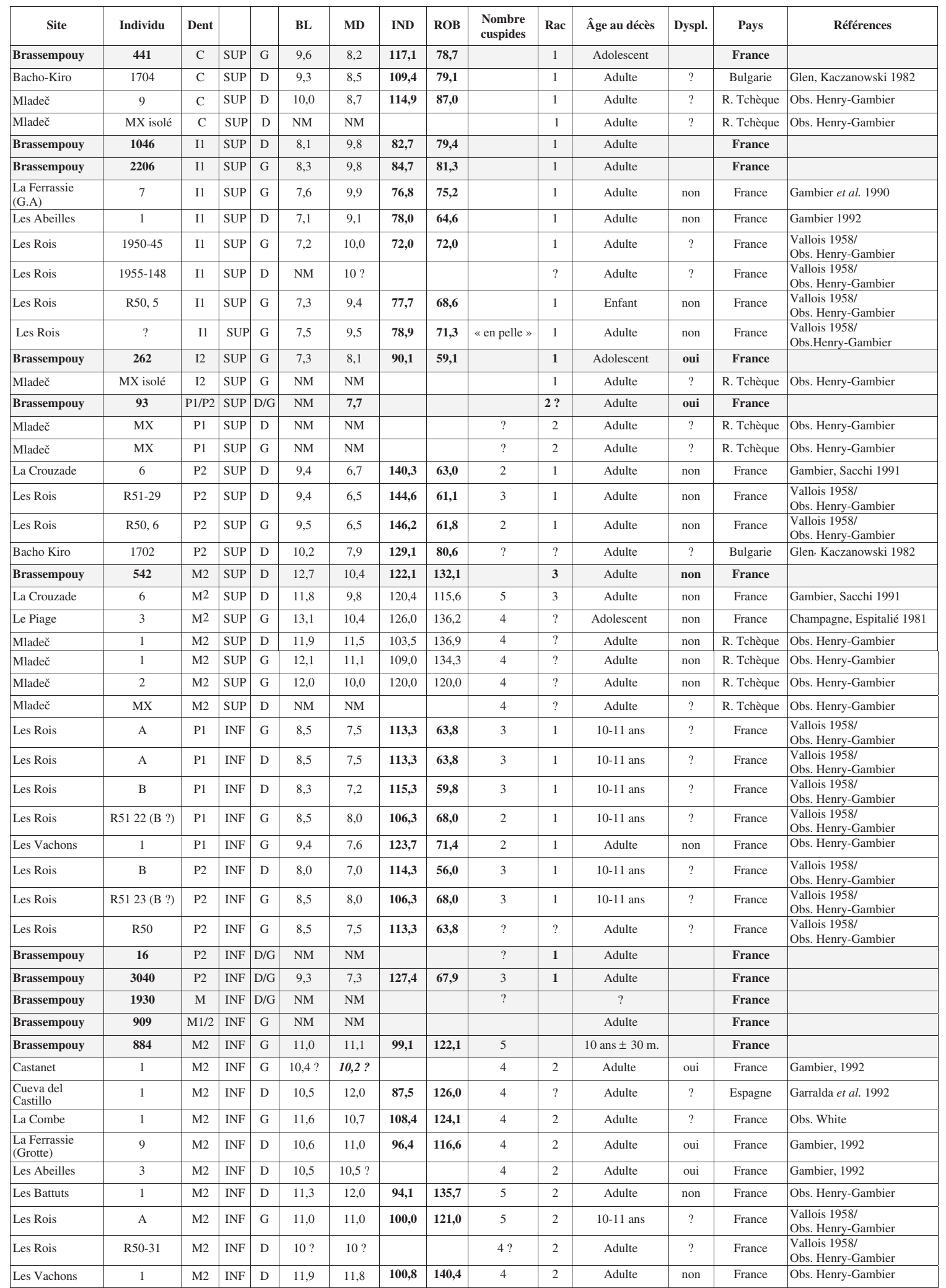

Tab. V - Europe, caractéristiques des dents permanentes aurignaciennes. $M D=$ diamètre mésio-distal de la couronne, $B L=$ diamètre bucco-lingual de la couronne, IND = indice de la couronne [BL/MD $\times 100]$, $R O B=$ robustesse de la couronne $=B L \times M D, R=$ Nbre de racines.

Tab. V-Europe, characteristics of Aurignacian permanent teeth. $M D=$ mesio-distal diameter of the crown, $B L=$ bucco-lingual diameter of the crown, IND = index of the crown [BL/MD $\times 100]$, $R O B=$ robustness of the crown $=B L \times M D, R=$ number of roots. 
protocole intégrant la loi de probabilité de la courbe de Student et la valeur centrée réduite ajustée (VCRA) adaptées au traitement des échantillons d'effectif faible. Selon cette méthode, avec un échantillon dont l'effectif est de trente, $95 \%$ de la variabilité de la population estimée est comprise dans l'intervalle $[(2,045$ x s) \pm moyenne $]$ et non entre $[(1,96 \times \mathrm{x} \mathrm{s}) \pm$ moyenne $]$ comme dans la majorité des travaux en paléoanthropologie. Avec un effectif de 10, l'équation devient $[(2,261 \mathrm{x} \quad \mathrm{s})$ \pm moyenne]. Les conséquences d'une telle approche sur les résultats des comparaisons entre des échantillons de fossiles humains de petit effectif sont évidentes. La méthode de la VCRA est présentée dans Maureille et al. (2001).

La valeur centrée réduite ajustée (tabl. VI, VII) varie de 0 (dans ce cas la valeur du spécimen étudié est égale à la moyenne de l'échantillon de comparaison) à plus ou moins l'infini. $95 \%$ de la variabilité de la population estimée à partir de l'échantillon de comparaison sont compris entre les valeurs +1 et -1 . Si la VCRA du fossile tombe entre -1 et +1 , elle s'intègre dans les limites de la variation estimée de la population de comparaison. Si elle est supérieure à 1 ou inférieure à -1 , elle est exclue de $95 \%$ de la variation estimée de la population de comparaison.

Après cette première étape de l'analyse, les dents de Brassempouy, dont au moins un paramètre était en dehors des $95 \%$ de la variabilité d'un groupe fossile, ont été traitées (Logiciel Statistica 6) par la méthode des ellipses équiprobables qui englobent $95 \%$ de la variabilité de l'échantillon étudié. Les ellipses ont été tracées dès lors que l'effectif de l'échantillon était d'au moins 10 . Un tel effectif est bien évidemment très faible au regard des normes admises par les statisticiens. Les groupes 4 et 5 ont été considérés. Un septième groupe constitué des hommes d'anatomie moderne du Paléolithique supérieur européen (Gravettien, Épigravettien, Solutréen, Badegoulien et Magdalénien) a été créé (cf. annexe II). Dans cet ensemble, un symbole distingue les Gravettiens, permettant de discuter leur position. Les Aurignaciens représentés par des dents isolées n'ont pas été intégrés dans les calculs, leur appartenance taxinomique étant sujette à caution mais leurs dents, dont celles de Brassempouy, ont été positionnées.

\begin{tabular}{|c|c|c|c|c|c|c|c|c|c|c|c|c|c|}
\hline \multirow{2}{*}{ Fossiles } & \multirow{2}{*}{ Variables } & \multirow{2}{*}{$\begin{array}{c}\text { Valeurs } \\
\text { individuelles } \\
\text { Brassempouy }\end{array}$} & \multicolumn{2}{|c|}{$\begin{array}{l}\text { H. actuels } \\
n^{\circ} 1\end{array}$} & \multicolumn{3}{|c|}{$\begin{array}{l}\text { H. actuels } \\
n^{\circ} 2\end{array}$} & \multicolumn{2}{|c|}{$\begin{array}{l}\text { H. modernes } \\
\text { (Gravettien) }\end{array}$} & \multicolumn{2}{|c|}{$\begin{array}{l}\text { H. modernes } \\
\text { Pal. moy. }\end{array}$} & \multicolumn{2}{|c|}{$\begin{array}{l}\text { Néandertaliens } \\
\text { Würm ancien }\end{array}$} \\
\hline & & & Inclus & Position & Inclus & Position & $\begin{array}{c}\text { Nombre } \\
\text { populations }\end{array}$ & Inclus & Position & Inclus & Position & Inclus & Position \\
\hline \multirow{4}{*}{$\begin{array}{l}\text { Abri Dubalen } \\
441 \text { CSUP }\end{array}$} & MD & 8,2 & NON & 0,74 & Oui & 0,25 & 178 & Oui & 0,11 & Oui & $-0,41$ & Oui & $-0,05$ \\
\hline & BL & 9,6 & NON & 1,20 & Oui & 0,92 & 116 & Oui & 0,40 & Oui & 0,21 & Oui & $-0,05$ \\
\hline & IND & 117,1 & Oui & 0,67 & & & & Oui & 0,42 & Oui & 0,41 & Oui & $-0,02$ \\
\hline & ROB & 78,7 & NON & 1,13 & & & & Oui & 0,27 & Oui & $-0,01$ & Oui & $-0,07$ \\
\hline \multirow{4}{*}{$\begin{array}{l}\text { Grotte des Hyènes } \\
1046 \text { I1SUP }\end{array}$} & MD & 9,8 & NON & 1,44 & Oui & 0,79 & 173 & Oui & 0,45 & Oui & $-0,41$ & Oui & 0,25 \\
\hline & BL & 8,1 & NON & 1,14 & Oui & 0,62 & 91 & Oui & 0,35 & Oui & 0,21 & Oui & 0,02 \\
\hline & IND & 82,7 & Oui & $-0,24$ & & & & Oui & $-0,19$ & Oui & 0,41 & Oui & $-0,26$ \\
\hline & ROB & 79,4 & NON & 1,63 & & & & Oui & 0,54 & Oui & $-0,01$ & Oui & 0,20 \\
\hline \multirow{4}{*}{$\begin{array}{l}\text { Grotte des Hyènes } \\
2206 \text { I1SUP }\end{array}$} & MD & 9,8 & NON & 1,48 & Oui & 0,82 & 173 & Oui & 0,47 & Oui & $-0,06$ & Oui & 0,28 \\
\hline & BL & 8,3 & NON & 1,42 & Oui & 0,83 & 91 & Oui & 0,60 & Oui & 0,09 & Oui & 0,30 \\
\hline & IND & 84,7 & Oui & $-0,06$ & & & & Oui & $-0,11$ & Oui & 0,08 & Oui & $-0,12$ \\
\hline & ROB & 81,3 & NON & 1,85 & & & & Oui & 0,68 & Oui & 0,04 & Oui & 0,35 \\
\hline \multirow{4}{*}{$\begin{array}{l}\text { Grotte des Hyènes } \\
262 \text { I2SUP }\end{array}$} & MD & 8,1 & Oui & 1,35 & Oui & 0,78 & 176 & Oui & 0,71 & Oui & $-0,25$ & Oui & 0,28 \\
\hline & $\mathrm{BL}$ & 7,3 & NON & 1,20 & Oui & 0,58 & 95 & Oui & 0,30 & Oui & 0,28 & Oui & $-0,79$ \\
\hline & IND & 90,1 & Oui & $-0,37$ & & & & Oui & $-0,39$ & Oui & 0,34 & NON & $-1,25$ \\
\hline & ROB & 59,1 & NON & 1,54 & & & & Oui & 0,65 & Oui & 0,16 & Oui & $-0,22$ \\
\hline \multirow{4}{*}{$\begin{array}{l}\text { Grotte des Hyènes } \\
3040 \text { P1INF }\end{array}$} & $\mathrm{MD}$ & 7,3 & Oui & 0,64 & Oui & 0,11 & 176 & Oui & $-0,13$ & Oui & $-0,30$ & Oui & $-0,07$ \\
\hline & BL & 9,3 & NON & 1,37 & Oui & 0,72 & 141 & Oui & 0,47 & Oui & 0,23 & Oui & 0,22 \\
\hline & IND & 127,4 & Oui & 0,66 & & & & Oui & 0,63 & NON & 1,03 & Oui & 0,21 \\
\hline & ROB & 67,9 & NON & 1,18 & & & & Oui & 0,15 & Oui & $-0,02$ & Oui & 0,07 \\
\hline \multirow{4}{*}{$\begin{array}{l}\text { Grotte des Hyènes } \\
542 \text { M2SUP }\end{array}$} & MD & 10,4 & Oui & 0,99 & Oui & 0,34 & 160 & Oui & 0,08 & Oui & $-0,06$ & Oui & $-0,04$ \\
\hline & BL & 12,7 & NON & 1,12 & Oui & 0,69 & 141 & Oui & 0,14 & Oui & 0,45 & Oui & 0,02 \\
\hline & IND & 122,1 & Oui & $-0,03$ & & & & Oui & 0,12 & Oui & 0,18 & Oui & 0,11 \\
\hline & ROB & 132,1 & NON & 1,30 & & & & Oui & 0,10 & Oui & 0,12 & Oui & $-0,04$ \\
\hline \multirow{4}{*}{$\begin{array}{l}\text { Grotte des Hyènes } \\
884 \text { M2INF }\end{array}$} & MD & 11,1 & Oui & 0,52 & Oui & 0,23 & 162 & Oui & $-0,18$ & Oui & 0,09 & Oui & $-0,39$ \\
\hline & $\mathrm{BL}$ & 11,0 & Oui & 0,99 & Oui & 0,35 & 140 & Oui & $-0,08$ & Oui & $-0,09$ & Oui & 0,02 \\
\hline & IND & 99,1 & Oui & 0,38 & & & & Oui & 0,13 & Oui & $-0,33$ & Oui & 0,40 \\
\hline & ROB & 122,1 & Oui & 0,83 & & & & Oui & $-0,15$ & Oui & $-0,01$ & Oui & $-0,21$ \\
\hline
\end{tabular}

Tabl. VI - Brassempouy - Dents permanentes : Valeurs centrées réduites ajustées en fonction des 5 échantillons de comparaison. Oui = la dent est incluse dans la variabilité du groupe de comparaison, NON = la dent n'est pas incluse dans la variabilité du groupe de comparaison. Oui ou NON en gras $=V C R A>$ ou $=$ à 0,95.

Table VI-Brassempouy - permanent teeth: Adjusted modified Z - score. Oui = the tooth is included in the variability of the comparative sample, $N O N=$ the tooth is not included in the variability of the comparative sample. Oui or NON in bold = Adjusted modified Z-score $>$ or $=$ to 0.95 . 
Pour les phalanges distales de la main, 10 mesures (longueur maximale, longueur articulaire, hauteurs et largeurs proximales, au milieu et distales de la diaphyse) et six indices correspondant à ces dimensions rapportées à la longueur articulaire ont été sélectionnés. Les phalanges de Brassempouy ont ensuite été comparées à celles d'une série d'hommes actuels (Musgrave 1973) et à celles, très rares, de groupes fossiles (hommes d'anatomie moderne du Gravettien et du Moustérien).

\begin{tabular}{|c|c|c|c|c|c|c|c|c|c|c|c|c|c|}
\hline \multirow{2}{*}{ Fossiles } & \multirow{2}{*}{ Variables } & \multirow{2}{*}{$\begin{array}{c}\text { Valeurs } \\
\text { individuelles } \\
\text { Brassempouy }\end{array}$} & \multicolumn{2}{|c|}{ H. actuels $n^{\circ} 1$} & \multicolumn{3}{|c|}{ H. actuels $n^{\circ} 2$} & \multicolumn{2}{|c|}{$\begin{array}{l}\text { H. modernes } \\
\text { (Gravettien) }\end{array}$} & \multicolumn{2}{|c|}{$\begin{array}{l}\text { H. modernes } \\
\text { Pal. moy. }\end{array}$} & \multicolumn{2}{|c|}{$\begin{array}{l}\text { Néandertaliens } \\
\text { Würm ancien }\end{array}$} \\
\hline & & & Inclus & Position & Inclus & Position & $\begin{array}{c}\text { Nombre } \\
\text { populations }\end{array}$ & Inclus & Position & Inclus & Position & Inclus & Position \\
\hline \multirow{4}{*}{$\begin{array}{l}\text { Grotte des Hyènes } \\
2879 \text { DM1SUP }\end{array}$} & MD & 7,5 & Oui & 0,27 & Oui & 0,40 & 5 & Oui & 0,03 & Oui & $-0,69$ & Oui & $-0,32$ \\
\hline & BL & 9,9 & NON & 1,14 & NON & 1,20 & 5 & Oui & 0,33 & Oui & 0,45 & Oui & 0,93 \\
\hline & IND & 132,0 & Oui & 0,45 & & & & Oui & 0,32 & NON & 1,05 & Oui & 0,81 \\
\hline & ROB & 74,3 & Oui & 0,79 & & & & Oui & 0,15 & Oui & $-0,20$ & Oui & 0,28 \\
\hline \multirow{4}{*}{$\begin{array}{l}\text { Grotte des Hyènes } \\
3625 \text { DM2SUP }\end{array}$} & MD & 8,4 & Oui & $-0,26$ & Oui & $-0,55$ & 5 & Oui & $-0,36$ & NON & $-1,39$ & Oui & $-0,71$ \\
\hline & $\mathrm{BL}$ & 10,0 & Oui & 0,34 & Oui & 0,33 & 5 & Oui & $-0,15$ & Oui & $-0,58$ & Oui & $-0,27$ \\
\hline & IND & 119,0 & Oui & 0,63 & & & & Oui & $-0,15$ & Oui & 0,36 & Oui & 0,62 \\
\hline & ROB & 84,0 & Oui & 0,02 & & & & Oui & $-0,17$ & Oui & $-0,94$ & Oui & $-0,65$ \\
\hline \multirow{4}{*}{$\begin{array}{l}\text { Grotte des Hyènes } \\
112 \text { DM1INF }\end{array}$} & MD & 8,4 & Oui & 0,47 & Oui & 0,47 & 5 & Oui & $-0,28$ & Oui & $-0,86$ & Oui & $-0,49$ \\
\hline & $\mathrm{BL}$ & 7,8 & Oui & 0,78 & Oui & 0,41 & 5 & Oui & 0,56 & Oui & 0,34 & Oui & 0,29 \\
\hline & IND & 92,9 & Oui & 0,35 & & & & Oui & 0,92 & Oui & 0,61 & Oui & 0,78 \\
\hline & ROB & 65,5 & Oui & 0,75 & & & & Oui & 0,15 & Oui & $-0,08$ & Oui & $-0,01$ \\
\hline \multirow{4}{*}{$\begin{array}{l}\text { Grotte des Hyènes } \\
69 \text { DM2INF }\end{array}$} & MD & 10,4 & Oui & 0,38 & Oui & 0,33 & 5 & Oui & 0,11 & Oui & $-0,36$ & Oui & 0,11 \\
\hline & BL & 9,8 & NON & 1,09 & Oui & 0,79 & 5 & Oui & 0,50 & Oui & 0,04 & Oui & 0,64 \\
\hline & IND & 94,2 & Oui & 0,97 & & & & Oui & 0,19 & Oui & 0,37 & Oui & 0,45 \\
\hline & ROB & 101,9 & Oui & 0,81 & & & & Oui & 0,27 & Oui & $-0,13$ & Oui & 0,35 \\
\hline
\end{tabular}

Tabl. VII - Brassempouy - Dents déciduales : Valeurs centrées réduites ajustées en fonction des 5 échantillons de comparaison (même légende que tabl. VI).

Table VII-Brassempouy-deciduous teeth: Adjusted modified Z-score. (same legend as table VI).

\section{ANALYSE MORPHOLOGIQUE DES DENTS DE LA GROTTE DES HYÈNES ET DE LA GALERIE DUBALEN}

\section{Niveau 2A}

Dent 69 (fig. 3-2) - Seconde molaire déciduale inférieure gauche : la couronne est complètement calcifiée. Un à deux millimètres de racine sont conservés. L'émail, légèrement fissuré, manque dans la partie distale de la face linguale. Cette altération relève d'un processus naturel. La surface occlusale est usée en cuvette et la dentine est totalement exposée sauf au niveau de la cuspide mésio-linguale (stade 2-3). En dépit de l'usure occlusale, on remarque que cette dent avait au moins quatre cuspides.

Les faces distale et mésiale de la couronne portent une facette d'usure liée au contact avec les dents adjacentes. La facette d'usure distale est large, sa présence indique que la première molaire permanente était fonctionnelle.

Le stade d'usure de la couronne et l'état de lyse des racines démontrent qu'il s'agit d'une dent proche de sa

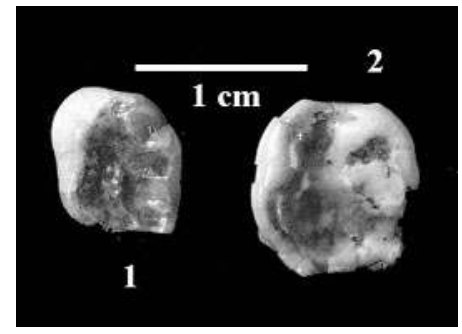

Fig. 3 - (1) DM1 inférieure gauche $n^{\circ} 112$,

(2) DM2 inférieure gauche $n^{\circ} 69$.

Fig. 3-(1) Left lower DM1 no.112, (2) left lower DM2 no.69. 
chute ou déjà tombée. Dans les populations actuelles cette perte intervient entre les stades 10 ans \pm 30 mois et 11 ans \pm 30 mois.

Dent 112 (fig. 3-1) - Première molaire déciduale inférieure gauche : la couronne et $1 \mathrm{~mm}$ de racine sont représentés. Un début de fissuration post mortem affecte l'émail. La surface occlusale est aplanie et la dentine est largement exposée sauf au niveau de la cuspide mésiolinguale (stade 3). Une large facette d'usure due au contact avec la DM2 entame la face distale et conduit à une réduction du diamètre mésio-distal. Le nombre de cuspides est indéterminable en raison de la forte usure occlusale. Toutefois, par comparaison avec des dents de populations actuelles au même stade d'attrition, la morphologie est analogue. Un étirement important de l'angle mésio-vestibulaire de la couronne, déterminant une bosse cervicale proéminente, est observable.

Comme la dent 69 et pour les mêmes raisons, cette dent était proche de la chute ou déjà tombée. Elle correspond à un âge dentaire comparable, soit 10 ans \pm 30 mois. Pour autant, les dents 69 et 112 n'appartiennent pas au même individu car les dimensions et la forme des facettes d'usure interproximales ne concordent pas.

Dent 93 (fig. 4) - Prémolaire supérieure : cette dent est représentée par la face vestibulaire de la couronne et par la moitié vestibulaire de la racine. La racine est longue, assez gracile. L'aspect de sa surface et celui des bords de la cassure indiquent une tendance au dédoublement. Une chambre pulpaire peu étendue et deux canaux radiculaires existaient. La face vestibulaire est sensiblement verticale et peu convexe suggérant que cette dent est une prémolaire supérieure. En revanche, il n'est pas possible de déterminer le côté.

La racine entièrement calcifiée concorde avec un âge dentaire d'au moins 15 ans \pm 36 mois, sans doute un adulte jeune si l'on considère le degré d'usure de la cuspide vestibulaire.

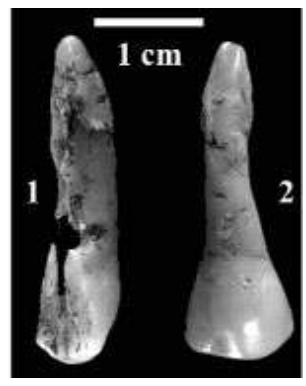

Fig. 4 - Prémolaire supérieure $n^{\circ} 93$, (1) face interne avec perforation, (2) face vestibulaire.

Fig. 4-Upper premolar no.93, (1) inner face with perforation, (2) buccal face.
Dent 16 (fig. 5) - Seconde prémolaire inférieure : ce fragment de prémolaire comprend la moitié linguale de la couronne et le tiers cervical de la racine. La cuspide linguale est aplanie sans que la dentine soit visible. Le sillon intercuspidien est à convexité linguale. Son éloignement du bord lingual de la couronne suggère que l'on est en présence d'une seconde prémolaire, mais le côté n'est pas déterminable.

Bien qu'il soit impossible de préciser l'état de calcification de la racine en raison de la destruction de la région apicale, la présence d'une facette d'usure interproximale, le stade d'usure de la cuspide linguale indiquent que cette dent était fonctionnelle et devait appartenir à un sujet adulte.

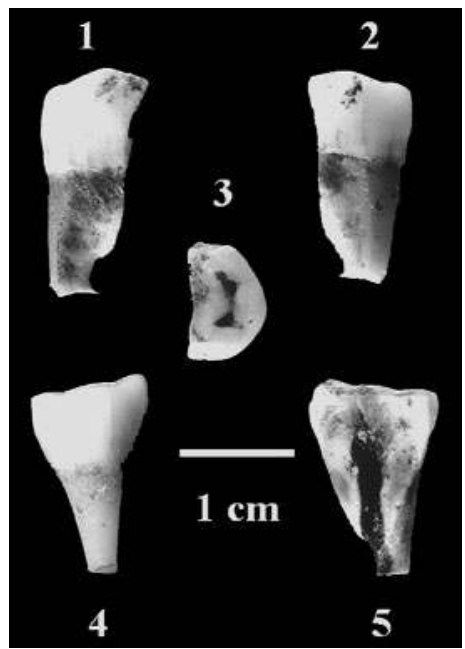

Fig. 5 - Seconde (?) prémolaire inférieure $(D / G ?) n^{\circ} 16$

$(1,2)$ face mésio/distale,

(3) face occlusale,

(4) face linguale,

(5) face interne.

Fig. 5-Second (?) right or left (?) lower premolar no. 16

$(1,2)$ mesio/distal face,

(3) occlusal face,

(4) lingual face,

(5) inner face.

Dent 884 (fig. 6) - Seconde molaire permanente inférieure gauche : c'est un germe dont les deux racines sont formées sur $6 \mathrm{~mm}$. La racine mésiale est plus développée dans le sens vestibulo-lingual que la racine distale.

La surface occlusale très ridulée avec de nombreux sillons secondaires présente cinq cuspides. Les deux

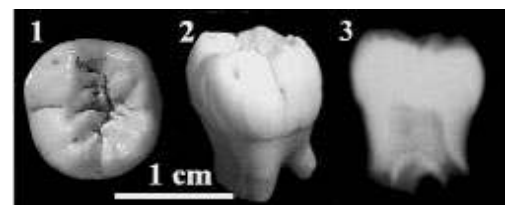

Fig. 6 - M2 inférieure gauche $n^{\circ} 884$, (1) face occlusale,

(2) face vestibulaire, (3) radiographie.

Fig.6-Left lower permanent M2 no.884. (1) occlusal face,

(2) buccal face, (3) radiograph. 
cuspides mésiales sont les plus développées. La cinquième cuspide, en position disto-vestibulaire, est réduite. La crête mésiale est peu développée et la fovea antérieure est petite. Il n'y a pas de fovea postérieure. La couronne est volumineuse avec une face vestibulaire bombée et un sillon vestibulaire assez profond.

Dans l'angle mésio-vestibulaire du tiers occlusal de la couronne on distingue une fossette. L'état de calcification de la racine correspond à un âge dentaire situé autour de 10 ans \pm 30 mois.

\section{Niveau 2C}

Dent 542 (fig. 7) - Seconde molaire permanente supérieure droite : lors de sa mise au jour cette dent était largement fendue, son exhumation a accéléré le processus de fracturation conduisant à sa séparation en trois fragments. La chambre pulpaire est de dimension moyenne, les trois racines entièrement calcifiées ne marquent aucune tendance à la fusion. Elles sont en outre très courtes. Des trois cuspides de la couronne, légèrement aplanies (stade d'usure 0-1), la cuspide linguale est la plus volumineuse. Il existe une facette d'usure mésiale. La forme trapézoïdale de la couronne, l'absence totale de fusion des racines et un début d'usure sur la face distale conduisent à identifier cette dent comme une seconde molaire supérieure. Cette usure distale qui prouve que la troisième molaire permanente était fonctionnelle et l'état de calcification des racines indiquent une dent appartenant à un sujet adulte relativement jeune.

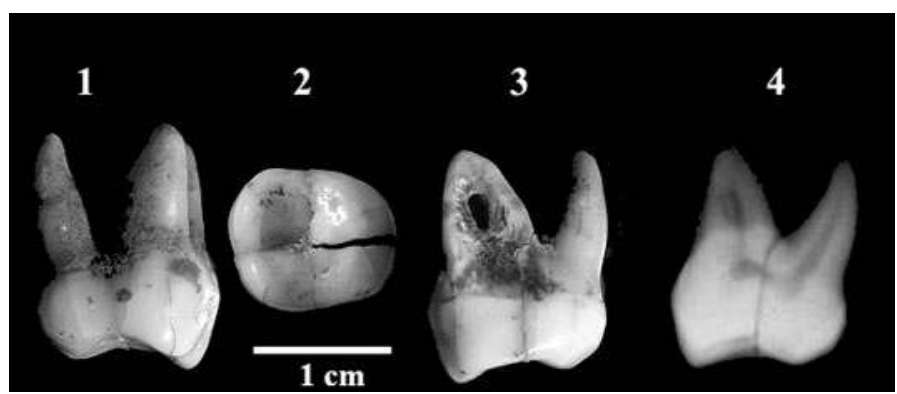

Fig. 7 - M2 supérieure droite $n^{\circ} 542$,

(1) face distale, (2) face occlusale,

(3) face mésiale avec racine mésio-vestibulaire percée, (4) radiographie.

Fig. 7-Right upper permanent M2 no.542,

(1) distal face, (2) occlusal face, (3) mesial face with perforated mesio-vestibular root, (4) radiograph.

Dent 1930 (fig. 8) - Fragment de molaire permanente inférieure : c'est une portion de couronne dont deux cuspides sont conservées. La cassure est ancienne et les bords sont émoussés. La face préservée de la couronne est verticale et les deux cuspides sont alignées parallèlement à celle-ci. Il s'agit certainement des deux cuspides linguales d'une molaire inférieure de rang (M1/M2/M3) indéterminable. L'émail est ridulé et de nombreux sillons secondaires convergent vers une fovea centrale. Le développement des deux cuspides laisse supposer une couronne volumineuse. L'absence d'usure de la surface occlusale et le stade de

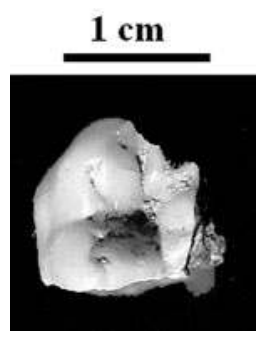

Fig. 8 - Fragment de molaire inférieure $n^{\circ} 1930$, face occlusale.

Fig. 8-Lower molar fragment no.1930 occlusal face. calcification de la racine formée sur 1 à $2 \mathrm{~mm}$ indiquent que l'on est en présence d'un germe dentaire correspondant à un âge dentaire de 4 ans \pm 12 mois (si M1), de 8 ans \pm 24 mois (si M2), de 15 ans \pm 36 mois (si M3).

Dent 2879 (fig. 9) - Germe de première molaire déciduale supérieure gauche : la couronne est totalement calcifiée et trois racines sont constituées sur 2 à $3 \mathrm{~mm}$. L'absence d'usure de la surface occlusale et le stade de calcification des racines correspondent à un âge dentaire situé autour du stade 1 an \pm 4 mois. Cette dent présente trois cuspides. Le protocône est particulièrement développé. Le paracône et le métacône sont moins bien individualisés. Une large fovea centrale existe. La face vestibulaire est très bombée. La bosse cervicale mésiovestibulaire est très proéminente (tuberculum molare). Les crêtes marginales mésiale et distale sont bien individualisées. Sur la crête distale, on observe une petite cuspide accessoire. La chambre pulpaire est vaste mais les racines ne marquent pas de tendance à la fusion.

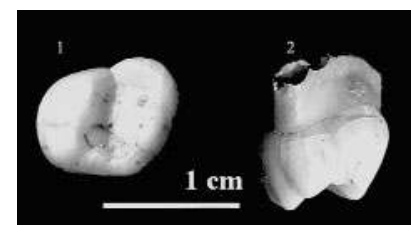

Fig. 9 - DM1 supérieure gauche $n^{\circ} 2879$,

(1) face occlusale, (2) face distale.

Fig. 9-Upper left DM1 no.2879, (1) occlusal face, (2) distal face. 
Dent 3040 (fig. 10) - Seconde prémolaire inférieure : la couronne est fissurée en raison de la dessiccation postfouille. L'extrémité de la racine manque (longueur totale de la racine $=8 \mathrm{~mm}$ ) à la suite d'une cassure ancienne.

La surface occlusale est usée (stade 1-2) et une fissure de la couronne existe. Les cuspides sont aplanies et la dentine est en partie exposée. Il semble cependant que cette dent avait deux cuspides principales et une cuspide accessoire en position mésio-linguale. La couronne de forme sensiblement rectangulaire évoque, en première analyse, une prémolaire supérieure. Cette conclusion est cependant invalidée par la convexité du sillon intercuspidien, par la forte convexité de la face vestibulaire ainsi que par la présence d'une troisième cuspide. Ces deux caractères et l'importance de la couronne plaident en faveur d'une seconde prémolaire inférieure, peut-être droite si on se base sur la concavité (en général la facette mésiale est concave) de l'une des facettes inter-proximales.
1

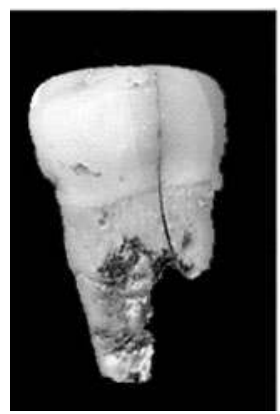

2

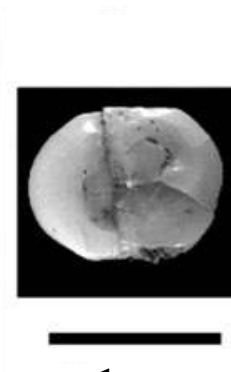

$1 \mathrm{~cm}$
3

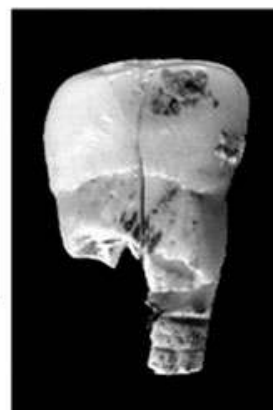

Fig. 10 - P2 inférieure (D?) $n^{\circ} 3040$,

(1) face distale? (2) face occlusale, (3) face mésiale?

Fig. 10-Right (?) lower second premolar no.3040,

(1) distal face (?), (2) occlusal face, (3) mesial face?

L'usure occlusale et la présence sur les faces mésiale et distale de la couronne de facettes d'usure interproximales prouvent que cette dent était fonctionnelle et avait achevé son éruption. Elle représente un sujet adulte. On remarque des stries verticales d'usure sur les facettes interproximales.

Dent 1046 (fig. 11) - Première incisive supérieure droite : un début de fissuration de l'émail et une altération d'origine chimique de l'extrémité de la racine sont observables. La face vestibulaire est légèrement convexe. La face linguale est peu concave. Les crêtes marginales et le tubercule lingual sont moyennement développés. Cette dent ne présente pas de morphologie « en pelle ».

Le bord incisif, usé en biseau, est rectiligne. Sur les faces distale et mésiale de la couronne, les facettes d'usure interproximales sont étroites et verticales. Sur la face vestibulaire, il n'y a aucune strie due à un objet en silex. La racine est courte et pyramidale. Cette dent appartient à un adulte.

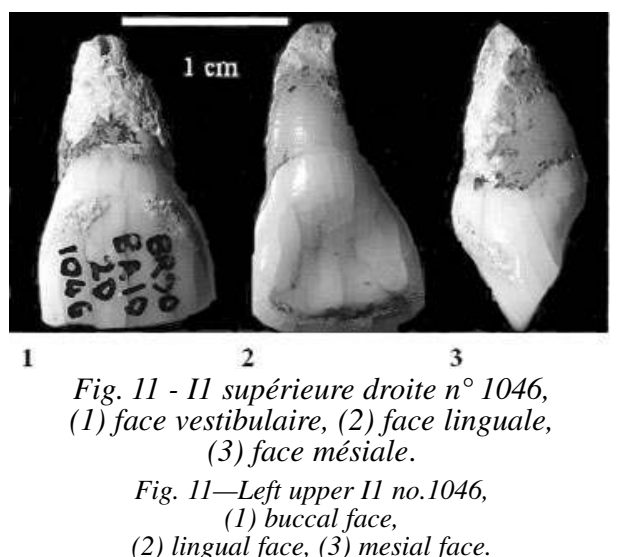

\section{Niveau 2E}

Dent 2206 (fig. 12) - Première incisive permanente supérieure gauche : cette dent est intacte. La racine, pyramidale, est très robuste et courte. Son apex est fermé. Le bord incisif de la couronne est usé en biseau (stade 1-2) et rectiligne. Des facettes d'usure interproximales sont visibles. La face vestibulaire est bombée. Le tubercule lingual est fort. Les crêtes marginales très marquées, en particulier la crête distale, délimitent une fosse linguale incisive profonde. Toutefois cette dent n'est pas en «pelle ». Sur la face vestibulaire, il n'y a aucune strie évidente due à un objet en silex.

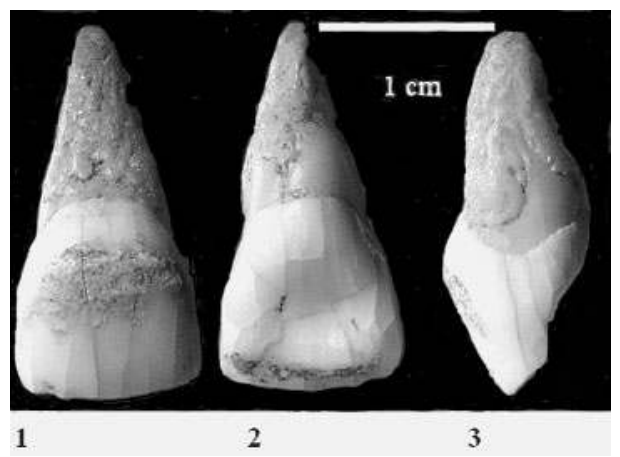

Fig. 12 - I1 supérieure gauche $n^{\circ} 2206$,

(1) face vestibulaire, (2) face linguale, (3) face distale.

Fig. 12-Left upper I1 no.2206,

(1) buccal face, (2) lingual face, (3) distal face.

L'état de calcification de la racine et le degré d'usure indiquent que cette dent appartient à un adulte. Cette dent n'est pas la dent symétrique de la dent 1046. Très proche morphologiquement, elle s'en distingue notamment au niveau des facettes d'usure interproximales. 
Dent 262 (fig. 13) - Seconde incisive supérieure gauche : cette dent est remarquablement conservée. L'apex de la racine est ouvert. L'usure de la couronne est nulle et le bord incisif est intact. L'état de calcification de la racine plaide en faveur d'un sujet adolescent. Cette conclusion concorde avec l'absence de trace d'usure qui indique que cette dent n'était fonctionnelle que depuis peu de temps. Le tubercule lingual et les crêtes marginales sont marqués. Ceci conduit à une fosse linguale profonde. Un sillon sépare le cingulum. Pour autant on ne peut parler de forme « en pelle ».

La racine est longue et aplatie dans le sens mésiodistal. Vers l'apex, sur la face distale se dessine un sillon peu marqué.

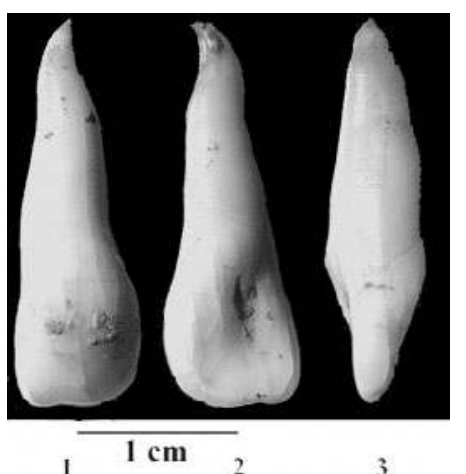

Fig. 13 - I2 supérieure gauche $n^{\circ} 262$, (1) face vestibulaire, (2) face linguale, (3) face mésiale.

Fig. 13-Left upper I2 no.262, (1) buccal face, (2) lingual face, (3) mesial face.

Dent 3625 (fig. 14) - Seconde molaire déciduale supérieure gauche : cette dent est représentée par la couronne. Les racines sont totalement lysées sauf à l'angle disto-vestibulaire. La surface occlusale est usée en cuvette et la dentine est totalement exposée. D'après les contours de la couronne, il semble que l'on soit en présence d'une dent à quatre cuspides dont celle distolinguale devait être réduite. La moitié supérieure de la face linguale, notamment au niveau de la cuspide mésiolinguale, est très convexe. Sur la face vestibulaire, le sillon intercuspidien est net.

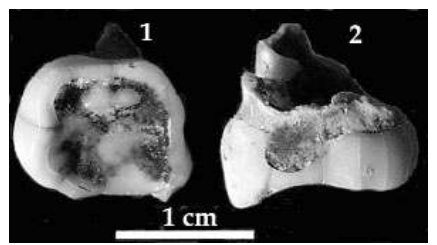

Fig. 14 - DM2 supérieure gauche $n^{\circ} 3625$,

(1) face occlusale, (2) face mésiale. Fig. 14-Left upper DM2 no.3625, (1) occlusal face, (2) mesial face.
L'état de résorption des racines et le stade d'usure occlusale indiquent une dent proche de sa chute voire déjà tombée, ce qui correspond à un âge dentaire de 10 ans \pm 30 mois.

\section{Couche I1}

Dent 441 (fig. 15) - Canine permanente supérieure gauche : cette dent est très bien conservée. La couronne est entièrement calcifiée, l'apex de la racine est ouvert. L'absence d'usure de la couronne et le stade de calcification de la racine concordent avec un sujet âgé d'au moins 10 ans \pm 30 mois mais n'ayant pas atteint l'âge adulte. La racine est aplatie dans le sens mésio-distal et ses deux faces présentent un sillon longitudinal marqué.

Le tubercule lingual est fort, la fossette distale est profonde et la crête marginale distale bien individualisée. Au-dessus du tubercule lingual, une petite crête se développe en direction de la cuspide. Un ou deux minuscules orifices s'ouvrent à la jonction de la crête marginale et du tubercule lingual.

L'état de conservation et la « fraîcheur » de cette dent ne sont pas sans évoquer ceux de la seconde incisive 262 provenant de la couche $2 \mathrm{E}$ du secteur S8 de la grotte des Hyènes qui communique avec l'abri Dubalen. Ces deux dents représentent en outre un stade analogue de développement de la denture. Elles pourraient appartenir au même individu.

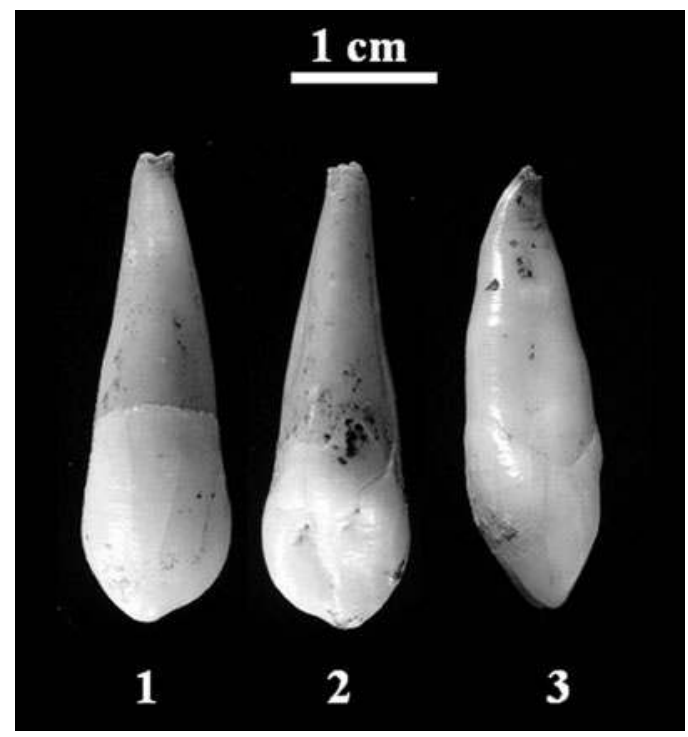

Fig. 15 - Canine supérieure gauche $n^{\circ} 441$, (1) face vestibulaire, (2) face linguale, (3) face distale.

Fig. 15-Left upper canine no.441, (1) buccal face, (2) lingual face, (3) distal face. 


\section{ANALYSE DES VESTIGES OSSEUX}

Grotte des Hyènes, couche 2A/2C - Phalanges distales de la main 302 et 344 (fig. 16) : les dimensions et la morphologie de ces deux phalanges distales de main sont compatibles avec celles de phalanges des doigts II, III, IV ou V. Si ces phalanges appartiennent au même individu, la phalange 344 , plus robuste, pourrait être une phalange du doigt III, la 302 plus gracile et dont la tubérosité distale est peu différenciée par rapport à la diaphyse serait une phalange du doigt $\mathrm{V}$.

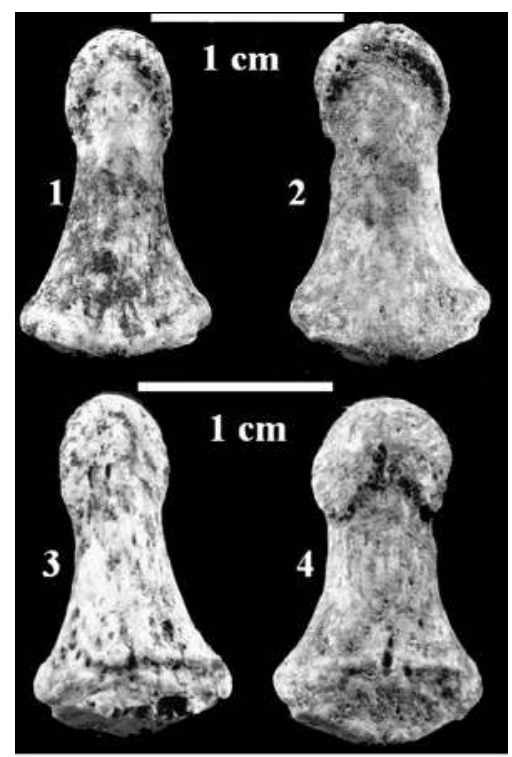

Fig. 16 - Phalanges distales de main $n^{\circ} 302$ et 344 (1) $n^{\circ} 302$ vue dorsale, (2) $n^{\circ} 344$ vue dorsale, (3) $n^{\circ} 302$ vue palmaire, (4) $n^{\circ} 344$ vue palmaire. Fig. 16-Distal hand phalanges (nos 302 and 344, (1) no.302, (2) no.344 dorsal view, (3) no.302, (4) no.344 palmar view.

Elles sont complètes. L'épiphyse proximale totalement fusionnée permet de les attribuer à un sujet adulte.

Sur la face palmaire de l'épiphyse distale, on distingue une zone plus irrégulière qui pourrait correspondre à l'insertion du muscle fléchisseur commun profond (m. flexor digitorum profundis).

La tubérosité (tuberositas phalangis distalis) de la phalange 344 est plus développée et plus large que celle de la phalange 302 .

Grotte des Hyènes, couche 2E - Fragment de crâne d'adulte (fig. 17-18) : il correspond à une petite portion de voûte située de part et d'autre de la suture sagittale. Le degré de courbure est très faible.
La suture sagittale (longueur $=28,9 \mathrm{~mm}$ ) est synostosée et en voie d'effacement du côté endocrânien, elle demeure bien visible sur la face exocrânienne.

Sur la face endocrânienne, on distingue le sinus sagittal qui est peu profond et une empreinte de vaisseau. $\mathrm{Ce}$ fragment pourrait correspondre à la région des pariétaux située quelques centimètres en arrière du bregma.

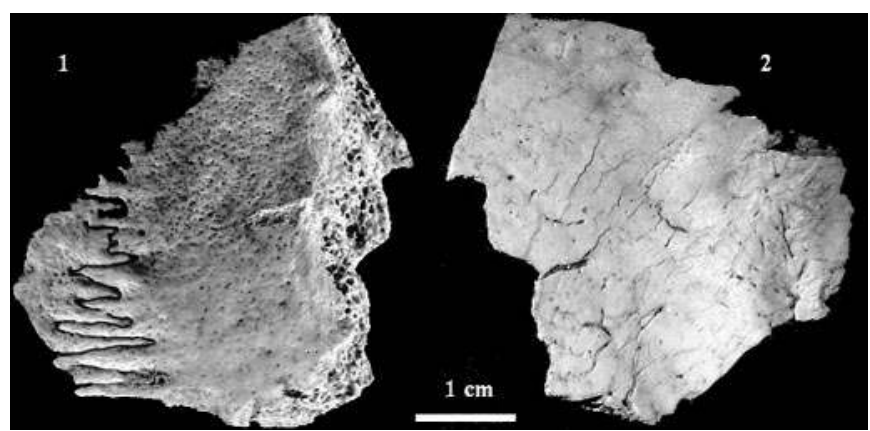

Fig. 17 - Fragment de crâne,

(1) vue exocrânienne, (2) vue endocrânienne.

Fig. 17-Cranial fragment, (1) exocranial view, (2) endocranial view.

L'épaisseur varie de $3,3 \mathrm{~mm}$ à $5,5 \mathrm{~mm}$. L'os est très bien conservé, les bords des cassures qui sont anciennes ne sont pas émoussés. Il est bien sûr impossible de dire si ce fragment de crâne représente un individu supplémentaire ou appartient à l'un des individus de la couche $2 \mathrm{E}$ identifié à partir des dents.

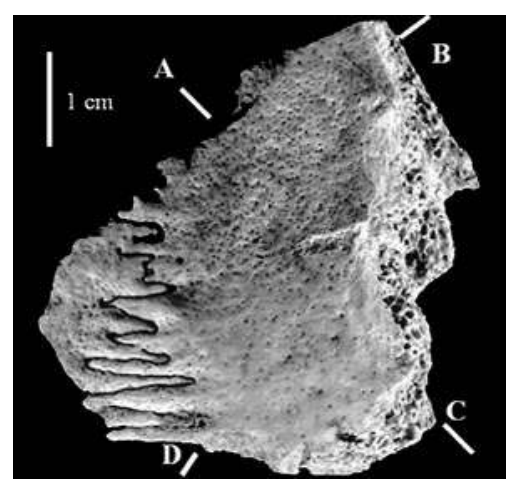

Fig. 18 - Fragment de crâne, vue exocrânienne, détail de la morphologie des cassures.

Fig. 18-Breakage pattern of the cranial fragment, exocranial view.

Grotte du Pape, couche F - Fragment de mandibule 909 (fig. 19) : ce fragment de corps mandibulaire gauche est long de $20 \mathrm{~mm}$. Il comprend une portion d'alvéole avec une molaire permanente encore en place. La face latérale du corps n'est pas conservée. Le 
bord inférieur est détruit et la surface de l'os est très érodée. L'altération est visiblement due à un processus chimique. Mais le polissage des surfaces évoque aussi un processus de transport et/ou l'action du ruissellement. Rappelons que dans la couche $2 \mathrm{~F}$, les os de faune peu abondants et mal conservés présentent des signes comparables d'altération. L'industrie osseuse y est également absente.

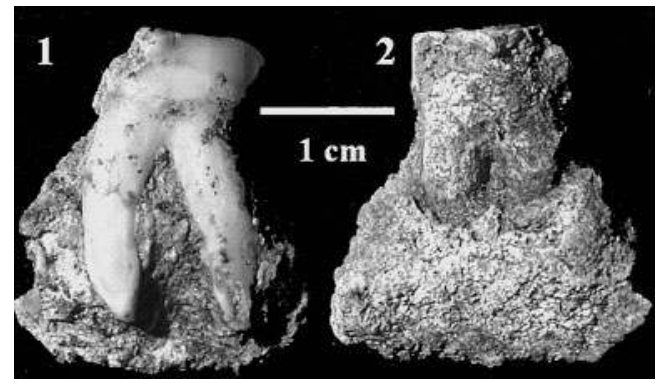

Fig. 19 - Fragment de mandibule $n^{\circ} 909$,

(1) vue médiale

(2) vue latérale.

Fig. 19-Mandible fragment no.909,

(1) medial view, (2) lateral view.

La couronne de la molaire se limite au quart vestibulo-distal. L'usure occlusale correspond à un stade 3-4. Sur la radiographie, on constate que l'apex des deux racines est fermé. Ce fragment de mandibule appartient à un adulte.

L'état de conservation de la mandibule et de la couronne empêche de statuer avec certitude sur le rang de la dent en place. Les dimensions de la couronne, la présence d'une facette d'usure distale excluent une troisième molaire. Il s'agit donc d'une première ou d'une seconde molaire.

\section{DÉNOMBREMENT}

Grotte des Hyènes - niveau $2 \mathrm{~A}$ : trois dents proviennent d'individus dont l'âge dentaire se situe autour du stade 10 ans \pm 30 mois $(884,69,112)$. Nous avons vu que les dents 69 et 112 n'appartiennent pas au même individu. En revanche, nous n'avons aucun élément pour admettre ou refuser le regroupement de la dent 884 avec l'une ou l'autre de ces deux dents.

Les dents 16 et 93 appartiennent à des adultes. Il est impossible de déterminer si elles représentent un ou deux adultes.

La couche $2 \mathrm{~A}$ a donc livré les restes d'au moins (NMI) deux enfants de 10 ans \pm 30 mois et d'un adulte.
Grotte des Hyènes - niveau 2C : il n'existe aucun élément permettant d'effectuer ou de refuser des regroupements entre les dents de ce niveau. Elles correspondent donc à au moins un adulte (dents 542, 1046 et 3040) et deux immatures (dents 1930, 2879) dont un de moins de 2 ans.

Il n'existe aucun indice permettant d'associer les deux phalanges isolées à l'un ou l'autre des adultes des niveaux $2 \mathrm{~A}$ ou $2 \mathrm{C}$ représentés par des dents ou, au contraire, d'en faire un sixième sujet.

Grotte des Hyènes - niveau 2E : il a livré trois dents qui représentent au moins trois individus si on se base sur le degré de calcification des racines, un adulte (2206), un adolescent (262) et un enfant (3625) de 10 ans \pm 30 mois. Il n'existe aucun élément permettant d'attribuer le fragment de crâne à l'adolescent ou à l'adulte.

Abri Dubalen - niveau I1 : la dent 441 correspond, comme la dent 262 du niveau 2E (secteur S8) de la grotte des Hyènes, à un adolescent. L'état de conservation et le stade de calcification des racines sont comparables. Comme le niveau I1 de Dubalen résulte d'un glissement de sédiments et de vestiges depuis les secteurs S8 et ouest de la grotte des Hyènes, il est possible que les dents 441 et 262 représentent le même adolescent.

Grotte du Pape - niveau 2F : le fragment de mandibule témoigne de la présence d'au moins un sujet adulte.

\section{PALÉOPATHOLOGIE}

Dysplasie de l'émail dentaire : deux dents, la prémolaire supérieure 93 et la seconde incisive supérieure 262 présentent des défauts de l'émail dentaire. Sur la dent 93 (fig. 4-2), il s'agit d'un défaut circulaire situé sur la face vestibulaire de la couronne à $1 \mathrm{~mm}$ du collet. Sur la dent 262 (fig. 13-3) un défaut linéaire apparaît sur la face mésiale à $1 \mathrm{~mm}$ du collet.

Des anomalies de l'émail dentaire ont été décrites chez les Néandertaliens (Henri-Martin 1923 ; Smith 1976 ; Tillier et al. 1989 ; Garralda, Vandermeersch 2000) ainsi que sur les dents de nombreux individus du Paléolithique supérieur d'Europe (Henry-Gambier 2001).

Ces irrégularités de la surface de l'émail sont en général mises en relation avec des anomalies mineures de l'amélogénèse. Dans le cas des dents 93 et 262, cette 
perturbation de l'amélogénèse aurait eu lieu avant l'âge de 6-7 ans auquel s'achève la minéralisation de la couronne de ces dents dans les populations actuelles.

Carie : sur la face mésiale de la seconde molaire déciduale 3625 , la surface d'usure liée au contact avec la DM1 est large et concave. Sur une surface sensiblement circulaire, concave de teinte gris foncé, l'émail a pratiquement disparu. Cette aspect pourrait correspondre à un début de carie (fig. 14). Une autre étiologie ne peut cependant pas être exclue. Les caries interproximales entre DM1 et DM2 seraient assez fréquentes chez les enfants actuels. Pour le Paléolithique supérieur nous n’en connaissons pas d'autre exemple.

Tartre : sur la seconde molaire supérieure 542 et la prémolaire supérieure 3040 , le tartre constitue un dépôt linéaire peu étendu et peu épais réparti sur la face linguale de la couronne à $2 \mathrm{~mm}$ au-dessus du collet.

Sur les trois incisives supérieures (1046, 262, 2206), l'épaisseur et l'extension du tartre sur la face vestibulaire sont plus importantes. Il constitue un dépôt qui débute 2 à $3 \mathrm{~mm}$ au-dessous du collet et s'interrompt $4 \mathrm{~mm}$ au-dessus du bord incisif (fig. 11, 12, 13).

Des dépôts de tartre ont été signalés sur les dents des populations du Paléolithique moyen (Henri-Martin 1923 ; Smith 1976 ; Trinkaus 1983 ; Tillier et al. 1989) ainsi que sur des dents des populations du Paléolithique supérieur. Le fait que de nombreuses dents du Paléolithique supérieur aient été nettoyées après leur découverte ne permet pas d'évaluer la fréquence de ces dépôts à cette époque.

Hypercémentose : la prémolaire supérieure 93 (fig. 4-1) et les deux premières incisives permanentes supérieures 1046 et 2206 (fig. 12, 13) ont des racines à surface irrégulière en raison d'appositions lamellaires irrégulières qui résultent vraisemblablement d'un processus d'hypercémentose.

Résorption alvéolaire : les racines de la molaire en place sur le fragment de mandibule 909 (fig. 19) trouvé dans la grotte du Pape sont mises à nu. Toutefois l'état de conservation de ce vestige ne permet pas de déterminer si cette exposition correspond à un processus de résorption alvéolaire ou à une érosion naturelle post mortem du bord alvéolaire.
Le fragment de crâne et les deux phalanges distales de main ne présentent aucune lésion d'ordre pathologique (fig. 16, 17).

\section{TRACES D'ACTION HUMAINE INTENTIONNELLE}

Quatre dents permanentes portent des traces liées à leur transformation en pendeloques (Henry-Gambier 2002 ; White et al. 2003 ; White, Henry-Gambier, sous presse).

Sur la dent 93 (fig. 4), on observe une perforation de la racine située sous le collet. Cette perforation mésiodistale a été précédée d'une phase d'amincissement dont témoignent les stries visibles sur l'une des faces. La racine et l'intérieur de la chambre pulpaire sont colorés en rouge.

La dent 16 (fig. 5) présente aussi des stries d'aménagement sur les faces " mésiale ou distale ${ }^{7}$ de la racine. Ces stries démontrent qu'une perforation était en cours de réalisation. Sur la moitié linguale de la face « mésiale ou distale » de la couronne, à hauteur de la facette d'usure interproximale, une altération de l'émail en rapport avec le façonnage de la racine de la dent est observable.

La racine mésio-vestibulaire de la dent 542 (fig. 7-3) est percée dans le sens mésio-vestibulaire. On distingue sur la face mésiale de la racine et de la couronne (au niveau de la facette d'usure mésiale) des stries consécutives au raclage qui a précédé la perforation. La dent est en outre légèrement ocrée, en particulier au niveau de la cavité pulpaire. Comme pour la dent 93 , il est possible que cette coloration résulte du contact avec le sédiment riche en ocre et non d'une coloration intentionnelle.

Les faces mésiale et distale de la racine de la dent 3040 (fig. 10) montrent des stries et des rainures qui prouvent que la racine de cette dent a aussi été travaillée. Ces traces se prolongent sur la couronne.

Le fragment de mandibule de la grotte du Pape est fortement dégradé avec une fissuration importante de l'os, caractéristique d'altérations liées à l'exposition aux agents atmosphériques (fig. 19). Cet état est en accord avec ce que l'on sait par ailleurs du mode de mise en place du niveau dont il provient. La faune de ce niveau a un état de conservation analogue.

Au contraire, le fragment de crâne (316) et les deux phalanges (302-344) de la grotte des Hyènes, comme les

7. Sur les dents qui ne peuvent être latéraliséees, les faces mésiale ou distale ne sont pas identifiables. 
dents isolées, ont un état de surface excellent (fig. 16, 17). La surface des deux phalanges est indemne de toute modification naturelle ou anthropique.

Les dimensions du fragment de crâne témoignent d'une fragmentation intense. Il ne présente ni strie de silex, ni trace d'origine animale. Il est limité par des cassures anciennes dont les bords ne sont pas émoussés (fig. 17, 18). Les segments AB, BC et CD sont biseautés sur la face exocrânienne. La lame externe a sauté par endroit ou est écaillée et le diploé est apparent. Le bord $\mathrm{AB}$ est rectiligne tandis que les bords $\mathrm{BC}$ et $\mathrm{CD}$ sont déchiquetés. Le bord $\mathrm{AD}$ est abrupt sans exposition du diploé (fig. 18). Aucune trace d'impact n'est visible mais nous ne disposons que d'un seul fragment. Les caractéristiques des cassures, bord biseauté, clivage entre diploé et arrachement de la lame externe, seraient typiques d'une fracturation survenue sur os frais (Boulestin 1998). Vu l'absence de marque imputable aux carnivores, l'hypothèse d'une fracturation d'origine anthropique se pose. Ceci constituerait un indice en faveur d'un traitement du cadavre.

\section{ANALYSE MORPHOLOGIQUE COMPARATIVE DES DENTS}

\section{Incisives permanentes supérieures}

Les incisives supérieures permanentes des Néandertaliens ont fréquemment une morphologie dite « en pelle » avec un tubercule lingual fort dédoublé ou non, une face linguale excavée et des crêtes latérales fortes et légèrement repliées. Un rétrécissement de la couronne à hauteur du collet est également signalé. La racine est longue et robuste (Patte 1959 ; Heim 1976 ; Smith 1976 ; Wolpoff 1979 ; Vandermeersch 1981 ; Wolpoff et al. 1981 ; Trinkaus 1983 ; Bermudez de Castro et al. 1999 ; Garralda, Vandermeersch 2000). À ces traits biologiques s'ajoute une forte usure occlusale souvent mise en relation avec un usage non alimentaire des dents antérieures.

Cette disposition « en pelle », connue dans les populations actuelles avec une fréquence variable, n'a pas été observée chez les populations moustériennes d'anatomie moderne comme Qafzeh et Skhul (Vandermeersch 1981 ; Tillier 1999). Elle est en revanche signalée sur une incisive aurignacienne (site des Rois, tabl. $V$ ) et certaines dents gravettiennes ont une morphologie « intermédiaire », voire en «pelle », comme le sujet immature de la double sépulture de la Grotte des Enfants en Italie.
Les incisives centrales supérieures (dents 1046 et 2206) et l'incisive latérale 262 de Brassempouy ne sont pas « en pelle ». Avec un fort tubercule lingual, des crêtes latérales bien individualisées, un rétrécissement coronaire au collet faible, une racine trapue et courte, leur morphologie est très proche des autres incisives aurignaciennes (tabl. V). Elles présentent la morphologie la plus fréquente dans les populations d'anatomie moderne actuelles et fossiles du Paléolithique moyen et supérieur.

\section{Canine permanente supérieure}

La canine supérieure des Néandertaliens a un tubercule lingual fort, prolongé parfois par une crête médiane. Cette morphologie caractérise aussi les populations d'anatomie moderne du Paléolithique moyen (Vandermeersch 1981). Les canines des hommes modernes du Paléolithique supérieur présentent une morphologie de la couronne comparable. Un épaississement fréquent du bord mésial est également signalé par Bailey (2002) sur les canines néandertaliennes. Il n'y a guère de références pour l'Aurignacien, les deux canines connues étant très usées (tabl. V). La canine de Brassempouy s'intègre donc dans la variabilité de ces groupes.

\section{Prémolaires supérieures}

Les prémolaires supérieures néandertaliennes se caractériseraient par une complexité importante de la face occlusale comparée aux dents de populations actuelles (Patte 1959 ; Heim 1976 ; Wolpoff 1979 ; Vandermeersch 1981 ; Trinkaus 1983 ; Garralda, Vandermeersch 2000 ; Bailey 2002). Une telle complexité n'a pas été décrite pour les dents de Qafzeh et de Skhul (Vandermeersch 1981 ; Tillier 1999). Les prémolaires supérieures aurignaciennes (tabl. V) ont deux cuspides et des crêtes mésiale et distale marquées. La surface occlusale est relativement simple. Cette morphologie caractérise aussi les prémolaires supérieures des populations d'anatomie moderne du Paléolithique supérieur et des populations actuelles.

L'état de conservation de la couronne de la dent 93 de Brassempouy limite les comparaisons.

\section{Seconde molaire permanente supérieure}

Les secondes molaires supérieures néandertaliennes ont quatre cuspides. Il n'y a pas de réduction de 
l'hypocône. Une cinquième cuspide et un tubercule de Carabelli sont aussi très fréquents et la surface occlusale est plus complexe que celle des dents des populations actuelles. Le taurodontisme des racines est également un trait fréquent chez les Néandertaliens (Patte 1959 ; Heim 1976 ; Wolpoff 1979 ; Vandermeersch 1981 ; Wolpoff et al. 1981 ; Trinkaus 1983 ; Garralda, Vandermeersch 2000 ; Bailey 2002). Des secondes molaires à trois cuspides ont cependant été décrites (Wolpoff et al. 1981 ; Bailey 2002).

Les hommes de Qafzeh et de Skhul ont des secondes molaires supérieures à quatre cuspides avec réduction de l'hypocône et présence d'un tubercule de Carabelli. Il n'y a pas de taurodontisme (Vandermeersch 1981 ; Tillier 1999).

La morphologie la plus fréquente des secondes molaires permanentes supérieures aurignaciennes est à quatre cuspides (tabl. V). La réduction de l'hypocône est parfois importante comme sur les secondes molaires supérieures de Mladeč 1 et 2 qui ont en outre une fosse disto-linguale. La seconde molaire de La Crouzade a une cinquième cuspide sur le bord distal. Aucune des dents aurignaciennes ne présente de taurodontisme. Dans les populations plus récentes du Paléolithique supérieur et dans les populations actuelles, les secondes molaires supérieures permanentes à quatre cuspides avec un hypocône réduit sont les plus fréquentes. La fréquence du tubercule ou du sillon de Carabelli y est variable.

Avec trois cuspides, la seconde molaire de Brassempouy n'a donc pas la forme la plus répandue, quelle que soit la population de référence.

\section{Prémolaires inférieures}

Dans les populations néandertaliennes et dans celles de Qafzeh-Skhul, les dents à deux cuspides dominent. Les dents à trois cuspides sont plus rares (P1 du Moustier 1, Régourdou). L'un des traits les plus discriminants des prémolaires néandertaliennes serait une surface occlusale avec de nombreux replis d'émail. Les prémolaires inférieures aurignaciennes sont à trois ou deux cuspides et la surface occlusale est simple (tabl. V). Dans les autres populations du Paléolithique supérieur et dans les populations actuelles, les dents à deux cuspides sont les plus fréquentes et la surface occlusale est simple.

La P2 inférieure (3040) de Brassempouy, avec deux cuspides principales et une possible troisième petite cuspide mésio-linguale, présente donc une morphologie connue dans chacune des populations de référence.

\section{Seconde molaire permanente inférieure}

Les secondes molaires inférieures néandertaliennes ont une surface occlusale souvent très ridulée avec de nombreux sillons secondaires. Elles ont le plus souvent cinq cuspides et la fovea antérieure est large. Dans l'échantillon de Qafzeh-Skhul, les dents à quatre cuspides disposées en croix sont les plus fréquentes (Vandermeersch 1981 ; Tillier 1999). Les Aurignaciens (tabl. V), comme les autres groupes du Paléolithique supérieur, ont des secondes molaires permanentes inférieures à quatre ou cinq cuspides. Les dents à cinq cuspides sont les plus fréquentes. La cinquième est généralement réduite. La fovea antérieure n'est jamais très large. C'est aussi le cas des secondes molaires sur la mandibule d'Oase 1 (Trinkaus et al. 2003)

La seconde molaire inférieure de Brassempouy paraît plus proche des secondes molaires des populations d'anatomie moderne du Paléolithique supérieur et actuelles que de celles des hommes modernes de QafzehSkhul ou de celles des Néandertaliens.

\section{Première molaire déciduale supérieure}

Les DM1 supérieures néandertaliennes ont une saillie cervicale forte sur la face vestibulaire (tuberculum molare) et trois cuspides. Les deux cuspides principales entourent une fovea centrale large. Métacône et hypocône sont en général à peine différenciés voire absents. Des cuspides accessoires peuvent exister (Tillier 1979, 1983). Les racines manifestent une tendance à la fusion (taurodontisme).

Les DM1 supérieures de Qafzeh, de forme quadrangulaire, ont quatre, trois ou deux cuspides. Métacône et hypocône sont réduits ou absents. Sur certaines DM1, une fovea antérieure et une fovea postérieure existent. La fovea centrale est plus ou moins vaste. Des cuspides accessoires peuvent aussi être présentes. Un tuberculum molare plus ou moins saillant existe. Aucun signe de fusion des racines n'est annoncé (Tillier 1999).

Nous n'avons pas de référence pour l'Aurignacien. Les DM1 supérieures des hommes modernes du Paléolithique supérieur offrent le même type de variations morphologiques que celles des populations moustériennes et actuelles (Henry-Gambier 2001). Avec deux cuspides principales, un métacône réduit, un tuberculum molare marqué, une fovea centrale profonde et une cuspide accessoire, la première molaire supérieure de 
Brassempouy s'insère dans la variabilité des différents groupes du Paléolithique moyen /supérieur et actuels.

\section{Seconde molaire déciduale supérieure}

Les DM2 supérieures des Néandertaliens sont de forme quadrangulaire et ont quatre cuspides. Les crêtes entre cuspides déterminent trois fosses (antérieure, centrale et postérieure). Une fossette ou un tubercule de Carabelli n'est pas rare. Les racines manifesteraient une tendance à la fusion (Tillier 1979, 1983).

Les enfants de Qafzeh ont des secondes molaires déciduales supérieures de forme quadrangulaire avec un étirement vestibulo-lingual plus ou moins marqué et quatre cuspides. La surface oclusale peut être ridulée avec de nombreux sillons secondaires. Une fovea centrale et une postérieure d'importance variable existent. La fovea antérieure n'est pas systématique. Une fossette ou un tubercule de Carabelli est fréquent (Tillier 1999).

Les DM2 supérieures des hommes d'anatomie moderne du Paléolithique supérieur sont aussi de forme quadrangulaire. Elles ont quatre cuspides et trois racines comme dans les populations actuelles. La fréquence du tubercule ou de la fossette de Carabelli est importante (Henry-Gambier 2001). Dans certains cas on observe une ridulation de la surface occlusale.

Les deux DM2 supérieures aurignaciennes ont une couronne quadrangulaire à quatre cuspides comme celle de Brassempouy (tabl. IV). L'usure de ces trois dents ne permet pas une description plus précise. On peut simplement constater, à partir des quelques caractères morphologiques observables, que les DM2 aurignaciennes ne se distinguent pas de celles des populations de référence fossiles ou actuelles.

\section{Première molaire déciduale inférieure}

Les DM1 inférieures néandertaliennes ont une asymétrie de la couronne. Cinq cuspides dont un hypoconulide réduit sont décrites. Une crête mésiale limite la fovea antérieure. La fovea centrale est plus ou moins vaste. La fovea postérieure n'est pas systématique. L'angle mésio-vestibulaire n'est pas toujours étiré en un tuberculum molare. Les racines manifestent une tendance à la fusion (Tillier 1979, 1983).

Les DM1 inférieures de Qafzeh sont de forme trapézoïdale à rectangulaire avec un étirement mésio- vestibulaire plus ou moins important et quatre cuspides principales. Les deux cuspides antérieures sont très rapprochées. Un hypoconulide vestigial peut être présent. La fovea antérieure est étroite, le bord mésial est épaissi en crête. La crête mésiale et la fovea postérieures sont inconstantes, la fovea centrale est large et profonde.

Les DM1 inférieures aurignaciennes sont rares (tabl. IV). La DM1 de Castillo dont l'usure est faible présente cinq cuspides. L'usure forte des autres DM1 ne permet pas de statuer sur le nombre de cuspides. Toutes, y compris celle de Brassempouy, se caractérisent par un étirement mésio-vestibulaire important et un espace étroit entre les cuspides antérieures.

Les hommes modernes du Paléolithique supérieur ont une DM1 avec des cuspides de petite taille, une fovea antérieure et parfois une fovea postérieure. L'angle mésiovestibulaire de la couronne est parfois étiré et les deux cuspides antérieures sont souvent étroitement accolées.

La DM1 inférieure de Brassempouy s'intègre donc dans la variabilité des différentes populations de référence actuelles et fossiles.

\section{Seconde molaire déciduale inférieure}

Les DM2 inférieures néandertaliennes ont cinq cuspides, parfois un tuberculum sextum, une fovea antérieure limitée par une crête mésiale et une fovea centrale. La surface peut être ridulée (Tillier 1979, 1983).

Les DM2 de Qafzeh, de forme rectangulaire à quadrangulaire, ont une saillie cervicale de la couronne plus ou moins marquée, cinq cuspides, une fovea centrale vaste et ridulée, une fovea antérieure bien individualisées. La fovea postérieure est inconstante (Tillier 1999).

Les DM2 inférieures aurignaciennes (tabl. IV) ont cinq cuspides, un tuberculum sextum peut être présent. L'hypoconulide est généralement très réduit. La surface occlusale est parfois ridulée. Une fovea antérieure plus ou moins vaste est aussi présente.

Cette morphologie est aussi la plus fréquente dans les autres populations du Paléolithique supérieur d'Europe et dans les populations modernes actuelles. La DM2 inférieure de Brassempouy est trop usée pour statuer avec certitude sur le nombre de cuspides. Sa forme quadrangulaire suggère plutôt une dent à cinq cuspides dont un hypoconulide très réduit. Elle s'intègre donc dans la variabilité des différentes populations de référence actuelles et fossiles. 


\section{ANALYSE MÉTRIQUE COMPARATIVE DES DENTS}

Les dents permanentes de Brassempouy se distinguent de celles des hommes actuels des groupes de référence par un diamètre $\mathrm{BL}$ et un diamètre $\mathrm{MD}$ plus grands. Les couronnes sont donc plus volumineuses et plus robustes. Leurs diamètres $\mathrm{MD}$ et $\mathrm{BL}$, ainsi que l'indice de robustesse, sont d'ailleurs en dehors de $95 \%$ de la variabilité de ces groupes ou situés à proximité de la limite supérieure de leur variabilité (tabl. VI).

Cette tendance caractérise aussi les dents déciduales. Elle est moins nette et seules deux variables (le diamètre BL de la DM1 et celui de la DM2 supérieures) sont exclues de la variabilité des groupes 1 et 2 d'hommes actuels (tabl. VII, fig. 20).

Excepté l'indice de la couronne de l'incisive latérale permanente (262) qui sort de la variabilité néandertalienne et celui de la première prémolaire inférieure (3040) qui est exclu de la variabilité des hommes de Qafzeh-Skhul, toutes les autres variables, quelle que soit la dent permanente considérée, sont comprises dans $95 \%$ de la variabilité des groupes fossiles de référence (tabl. VI).

La même remarque s'applique aux dents déciduales, pour lesquelles seul l'indice de la couronne de la DM1 supérieure (2879) et le diamètre MD de la DM2 supérieure (3625) sortent de la variabilité des hommes de QafzehSkhul (tabl. VII, fig. 21).

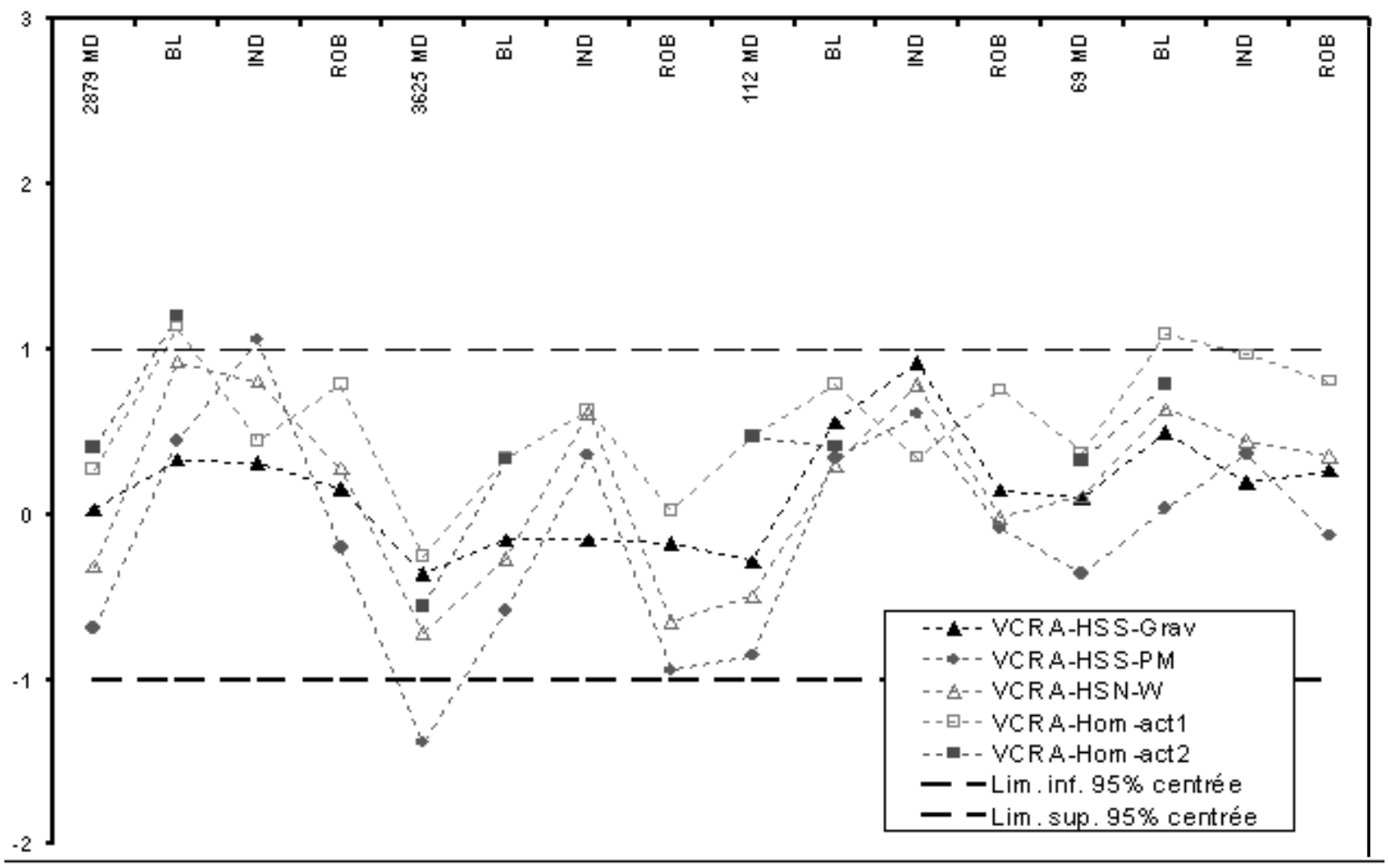

Fig. 20 - Dents déciduales : représentation graphique des valeurs centrées réduites ajustées. VCRA-HSS-grav = valeur centrée réduite ajustée des dents de Brassempouy par rapport à la variabilité des hommes modernes du Gravettien européen,

VCRA-HSS-PM = valeur centrée réduite ajustée des dents de Brassempouy par rapport à la variabilité des hommes d'anatomie moderne du Paléolithique moyen, VCRA-HSN-W = valeur centrée réduite ajustée des dents de Brassempouy par rapport à la variabilité des Néandertaliens würmiens,

VCRA-Hom-actl = valeur centrée réduite ajustée des dents de Brassempouy par rapport à la variabilité de l'échantillon d'hommes actuels $n^{\circ} 1$,

VCRA-Hom-act 2 = valeur centrée réduite ajustée des dents de Brassempouy par rapport à la variabilité de l'échantillon d'hommes actuels $n^{\circ} 2$.

Fig. 20-Brassempouy-Deciduous teeth: VCRA = Adjusted modified Z-score, HSS-grav. = Gravettian Homo sapiens sapiens from Europe, HSS-PM = Middle Palaeolithic Homo sapiens sapiens, $H S N-W=$ Würmian Neanderthals, Hom-act 1 and 2 = extant Homo sapiens sapiens. 
La majorité des dents de Brassempouy a donc des dimensions coronaires qui s'intègrent dans la variabilité des groupes fossiles (Néandertaliens et hommes d'anatomie moderne du Paléolithique moyen et supérieur) utilisés dans cette étude.

Toutefois, la comparaison avec les dents aurignaciennes (tabl. IV, $V$ ) et l'analyse des VCRA (tabl. VI, VII) de chaque dent de Brassempouy par rapport aux groupes fossiles gravettiens et moustériens de référence permet de les positionner dans la variabilité de ces groupes.

- Les incisives permanentes centrales supérieures (1046, 2206) ont, comme les autres incisives aurignaciennes, un diamètre $\mathrm{BL}$ petit par rapport au diamètre $\mathrm{MD}$ mais leur robustesse est légèrement supérieure. Comparé aux dents des hommes d'anatomie moderne du Paléolithique moyen, le diamètre BL est grand par rapport au diamètre MD. L'ensemble des valeurs centrées réduites ajustées (VCRA) montre que les deux incisives de Brassempouy sont plus éloignées des incisives des Gravettiens que de celles des hommes du
Paléolithique moyen. La dent 1046 apparaît alors plus proche de celle des Néandertaliens tandis que la dent 2206 est plus proche de celles du groupe d'anatomie moderne Qafzeh- Skhul.

- L'incisive permanente latérale 262 se caractérise aussi par un diamètre MD grand par rapport au diamètre BL. Il n'y a pas d'autre référence pour l'Aurignacien ancien.

Elle se distingue clairement des incisives latérales des Néandertaliens puisque son diamètre BL est proche de la limite inférieure de la variabilité néandertalienne, déterminant un indice de la couronne qui sort de leur variabilité. Comparée aux incisives du groupe gravettien, la dent 262 a aussi un diamètre BL petit par rapport au diamètre MD. En revanche, il est proportionnellement plus grand que celui des I2 supérieures du groupe Qafzeh-Skhul.

Les VCRA indiquent que la dent 262 est plus proche des I2 du groupe Qafzeh-Skhul que de celles des Gravettiens.

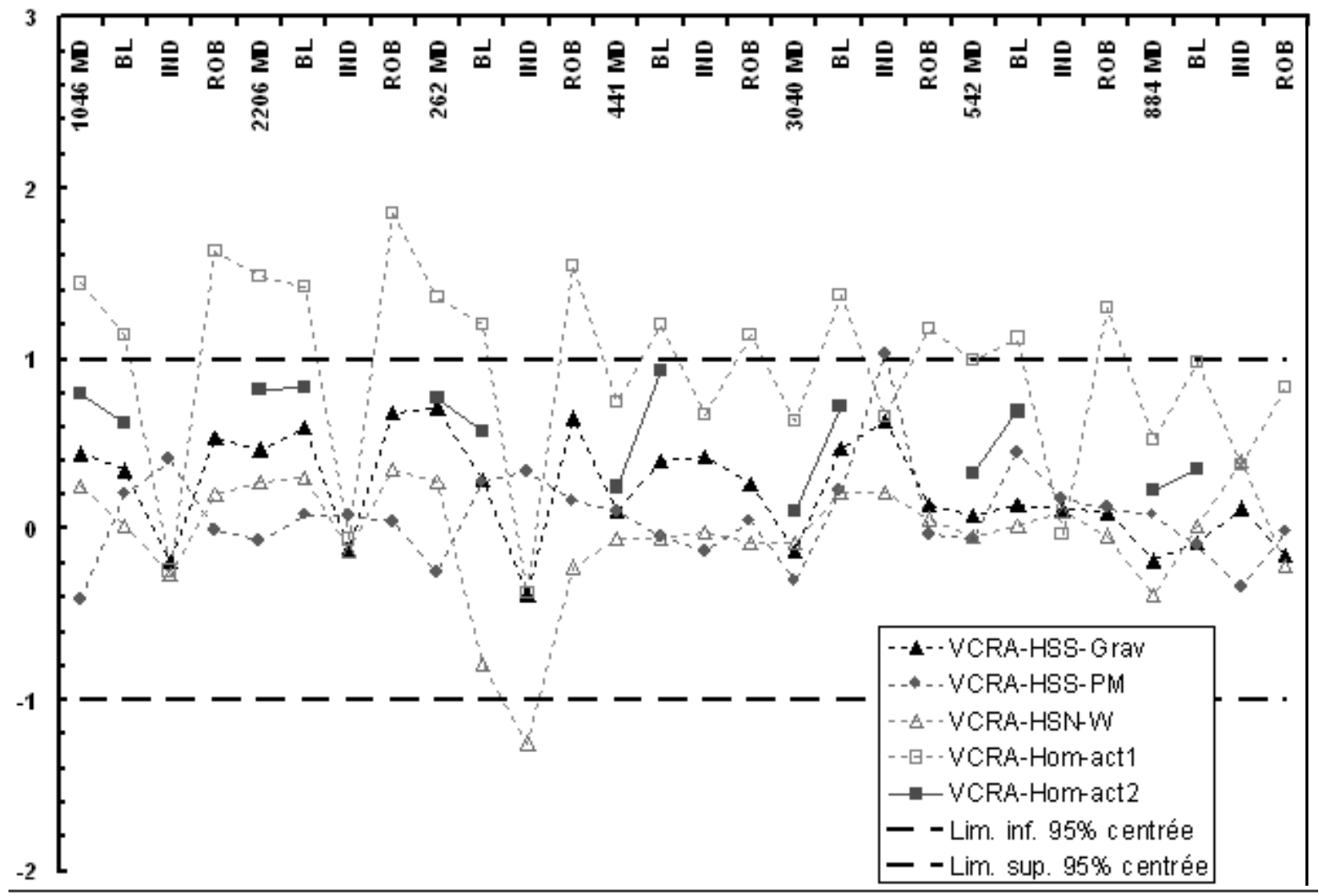

Fig. 21 - Dents permanentes : représentation graphique des valeurs centrées réduites ajustées (même légende que fig. 20). Fig. 21-Brassempouy-Deciduous teeth: VCRA = Adjusted modified Z-score (same legend as fig. 20). 
La zone de chevauchement des ellipses équiprobables des groupes fossiles est réduite (fig. 22). Les groupes du Paléolithique moyen (hommes d'anatomie moderne et Néandertaliens) se distinguent nettement de ceux du Paléolithique supérieur dont les diamètres BL et MD sont plus faibles pour la majorité des individus.

L'incisive 262, seule I2 aurignacienne mesurable, se situe dans la zone de chevauchement avec celles de Pataud 1 (Gravettien), de Vado All'Arancio 1 et de Romito 4 (Épigravettien récent).

- La canine permanente supérieure (441) a un diamètre $\mathrm{BL}$ élevé par rapport au diamètre $\mathrm{MD}$. C'est également le cas de deux canines supposées aurignaciennes (Mladeč et Bacho Kiro). Cependant la canine de Brassempouy est moins robuste.

La comparaison avec les canines du groupe gravettien montre que le diamètre $\mathrm{BL}$ est proportionnellement plus grand que le diamètre MD ; celle avec les hommes d'anatomie moderne (QafzehSkhul) indique que c'est le diamètre MD qui est le plus grand.

Les VCRA montrent que cette dent est plus proche des canines du groupe néandertalien que de celles des hommes d'anatomie moderne du Paléolithique moyen et supérieur, les Gravettiens étant les plus éloignés.

- La prémolaire supérieure (93) a un diamètre MD qui est supérieur aux diamètres MD de la série des dents aurignaciennes (tabl. V). Le diamètre BL n'a pu être estimé car la couronne est incomplète. Avec une valeur de $7,4 \mathrm{~mm}$, le diamètre $\mathrm{MD}$ est comparable au diamètre moyen des P1 des Gravettiens, des Néandertaliens et des hommes d'anatomie moderne du Paléolithique moyen (la différence est plus marquée avec ces derniers).

Il est en revanche nettement supérieur au diamètre MD des P2 de ces mêmes groupes.

Il est donc possible que cette dent soit une P1. Toutefois, en raison de la variabilité individuelle, il est plus prudent de ne pas conclure.

- La seconde prémolaire inférieure (3040) se caractérise, comme les deux autres prémolaires aurignaciennes (Les Rois et Bacho Kiro), par un diamètre BL supérieur au diamètre $\mathrm{MD}$ et sa robustesse est comparable. Quel que soit le groupe fossile de référence, le diamètre $\mathrm{BL}$ de cette dent reste supérieur au diamètre MD et l'indice de la couronne est élevé. Le développement bucco-lingual est marqué.

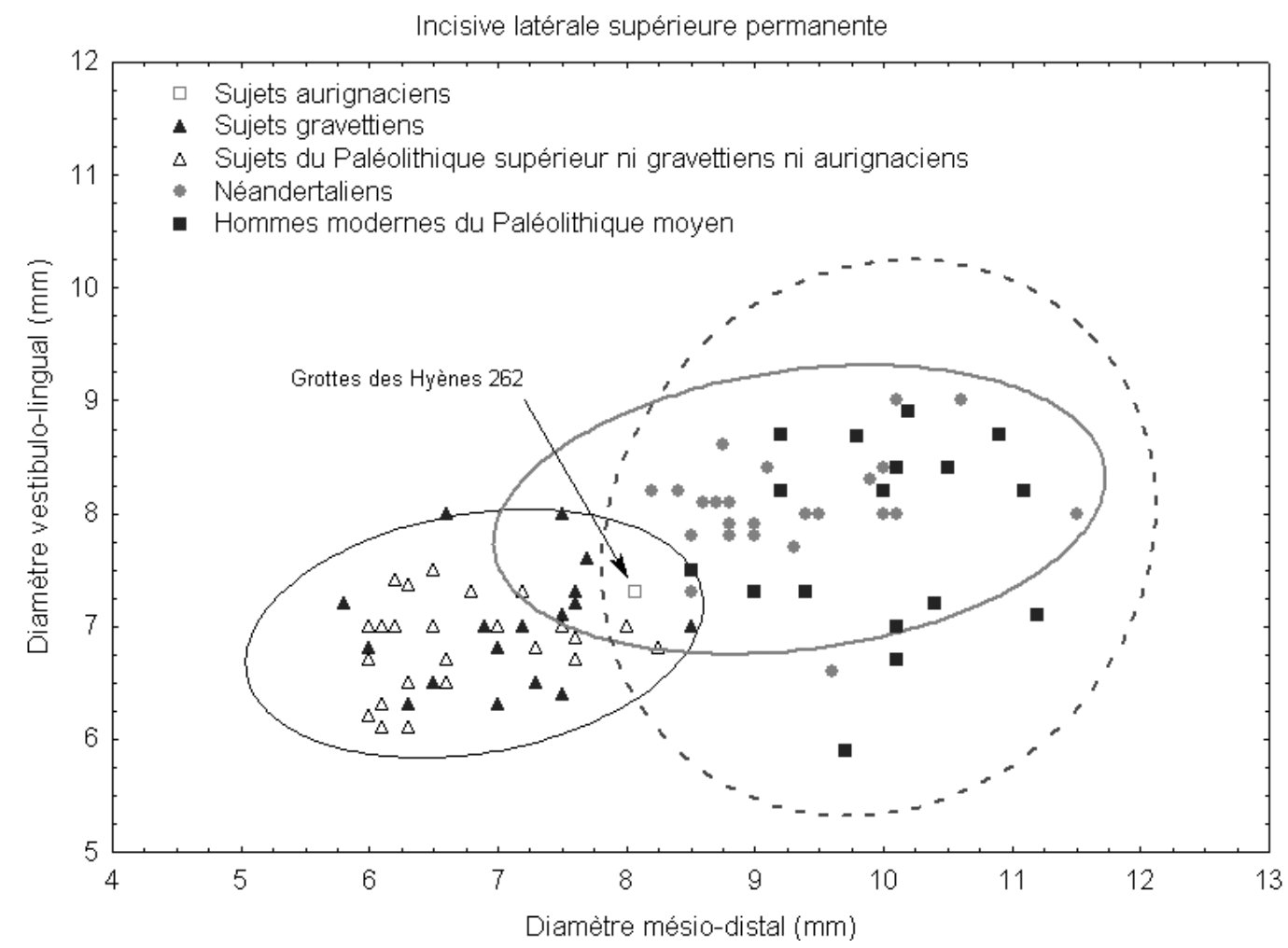

Fig. 22 - I2 permanente supérieure : ellipses équiprobables à $95 \%$ des échantillons de comparaison et position des Aurignaciens.

Fig. 22-Upper permanent I2: bivariate scatter plots. 
Les VCRA placent cette dent plus loin des prémolaires gravettiennes que de celles des fossiles du Paléolithique moyen et c'est avec les prémolaires des Néandertaliens que l'écart est le plus faible.

L'étude des ellipses équiprobables apporte des renseignements complémentaires (fig. 23). La zone de chevauchement de la variabilité de chaque groupe est très grande et la dent de Brassempouy se situe dans cet espace. Elle n'est plus exclue de la variabilité des hommes modernes du Paléolithique moyen. Toutefois, son diamètre BL est supérieur à celui de cinq des neuf sujets de ce groupe. La variabilité des hommes modernes du Gravettien est forte et le diamètre MD des prémolaires est, pour une majorité de sujets, supérieur à celui des hommes du Paléolithique supérieur récent. Les quatre dents aurignaciennes sont groupées. Deux dents, Isturitz 82 (Magdalénien supérieur) et Vergisson (NN4) qui est attribuée à un Néandertalien sortent de la variation des groupes fossiles.
- La seconde molaire permanente supérieure (542) offre un diamètre $\mathrm{BL}$ supérieur au diamètre $\mathrm{MD}$ comme les autres dents aurignaciennes et se situe, pour la robustesse, parmi les dents aurignaciennes les plus robustes.

Quel que soit le groupe fossile de comparaison, le développement en largeur (BL) de cette dent est plus important que son allongement.

Les VCRA indiquent que cette dent est plus proche de celles des Néandertaliens que de celles des hommes modernes du Paléolithique moyen et du Gravettien, l'écart le plus grand étant avec les dents des hommes d'anatomie moderne de Qafzeh-Skhul.

- Le germe de seconde molaire permanente inférieure (884) a un diamètre MD très faiblement supérieur au diamètre BL. La couronne de cette dent est presque carrée. Les autres M2 inférieures aurignaciennes se caractérisent aussi par une tendance à l'égalité des deux diamètres, mais elles sont en majorité plus robustes.

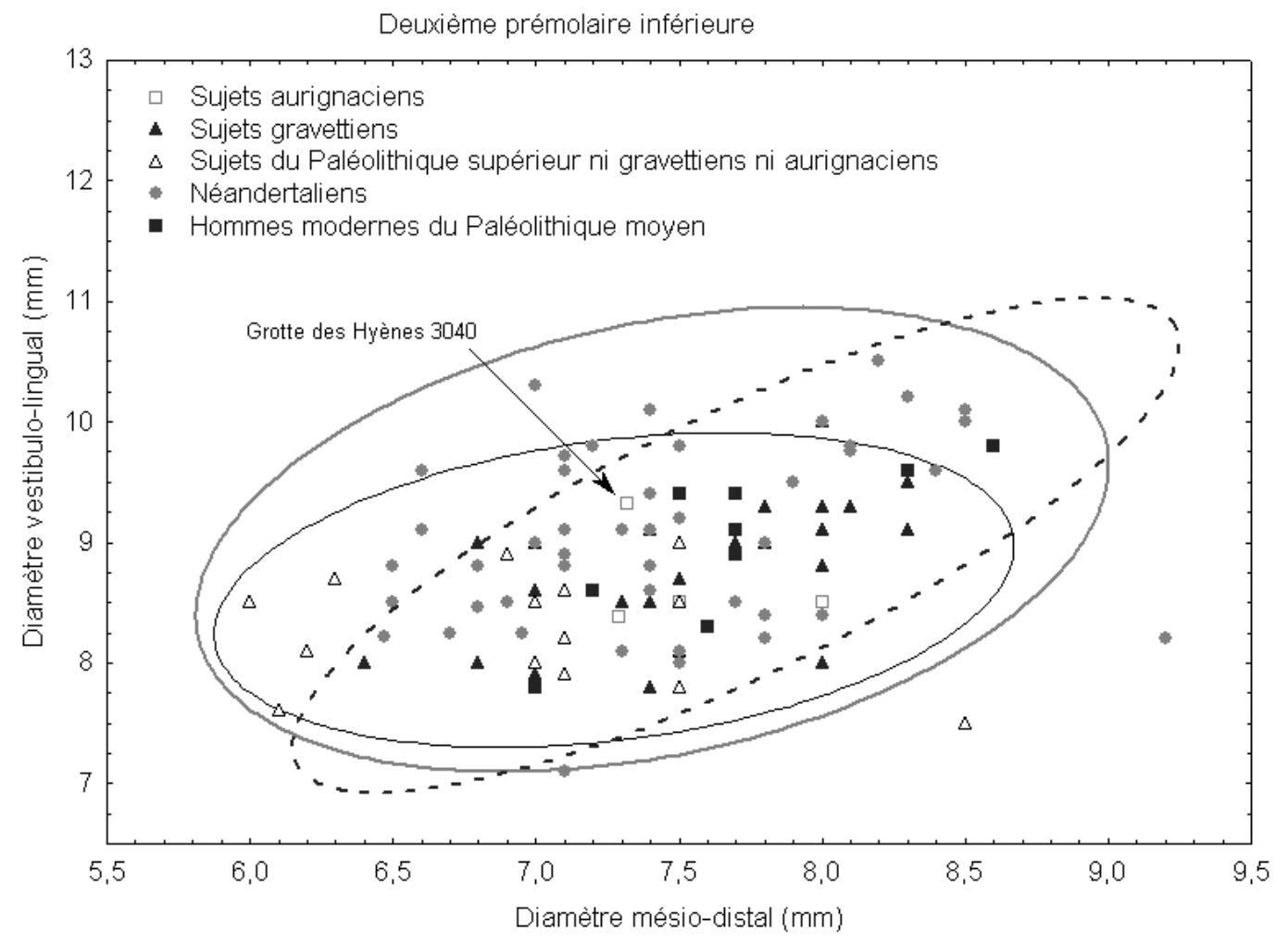

Fig. 23 - P2 inférieure: ellipses équiprobables à $95 \%$ des échantillons de comparaison et position des Aurignaciens.

Fig. 23-Lower P2: bivariate scatter plots. 
Comparé aux dents des Gravettiens et des Néandertaliens, son diamètre MD est petit par rapport au diamètre BL (allongement de la couronne plus faible). En revanche, le diamètre $\mathrm{MD}$ est plus grand que le diamètre BL des hommes d'anatomie moderne du Paléolithique moyen déterminant un indice de la couronne plus faible (allongement MD de la couronne plus important).

Les VCRA placent cette seconde molaire inférieure plus près des dents des Gravettiens et des hommes d'anatomie moderne du Paléolithique moyen que des dents du groupe néandertalien.

- La première molaire déciduale supérieure (2879) se caractérise par un diamètre BL supérieur au diamètre MD indiquant un grand développement bucco-lingual de la couronne. Il n'y a pas d'autre référence pour l'Aurignacien ancien.

Comparée aux DM1 des autres groupes fossiles, la dent de Brassempouy apparaît toujours plus large avec un diamètre $\mathrm{BL}$ important par rapport au diamètre $\mathrm{MD}$. La différence est nette avec les dents des hommes d'anatomie moderne du Paléolithique moyen car l'indice de la couronne est au-dessus de la limite supérieure de la variabilité de ce groupe.

Les VCRA montrent que la dent de Brassempouy est plus proche des DM1 supérieures des Gravettiens que de celles des groupes du Paléolithique moyen et parmi ces derniers, la dent de Brassempouy est plus proche des Néandertaliens.

La zone de chevauchement de la variabilité de chaque groupe fossile est très grande et la dent de Brassempouy se situe dans cet espace (fig. 24). Elle se place parmi les dents dont le diamètre BL est le plus grand.

- La seconde molaire déciduale supérieure (3625) a un diamètre BL supérieur au diamètre MD. L'indice de la couronne est élevé. Elle est moins robuste que la dent de Ksar'Aqil, son diamètre MD étant plus faible.

Comparée aux DM2 supérieures des groupes fossiles, cette dent se caractérise toujours par un diamètre $\mathrm{BL}$ proportionnellement plus grand que le diamètre $\mathrm{MD}$

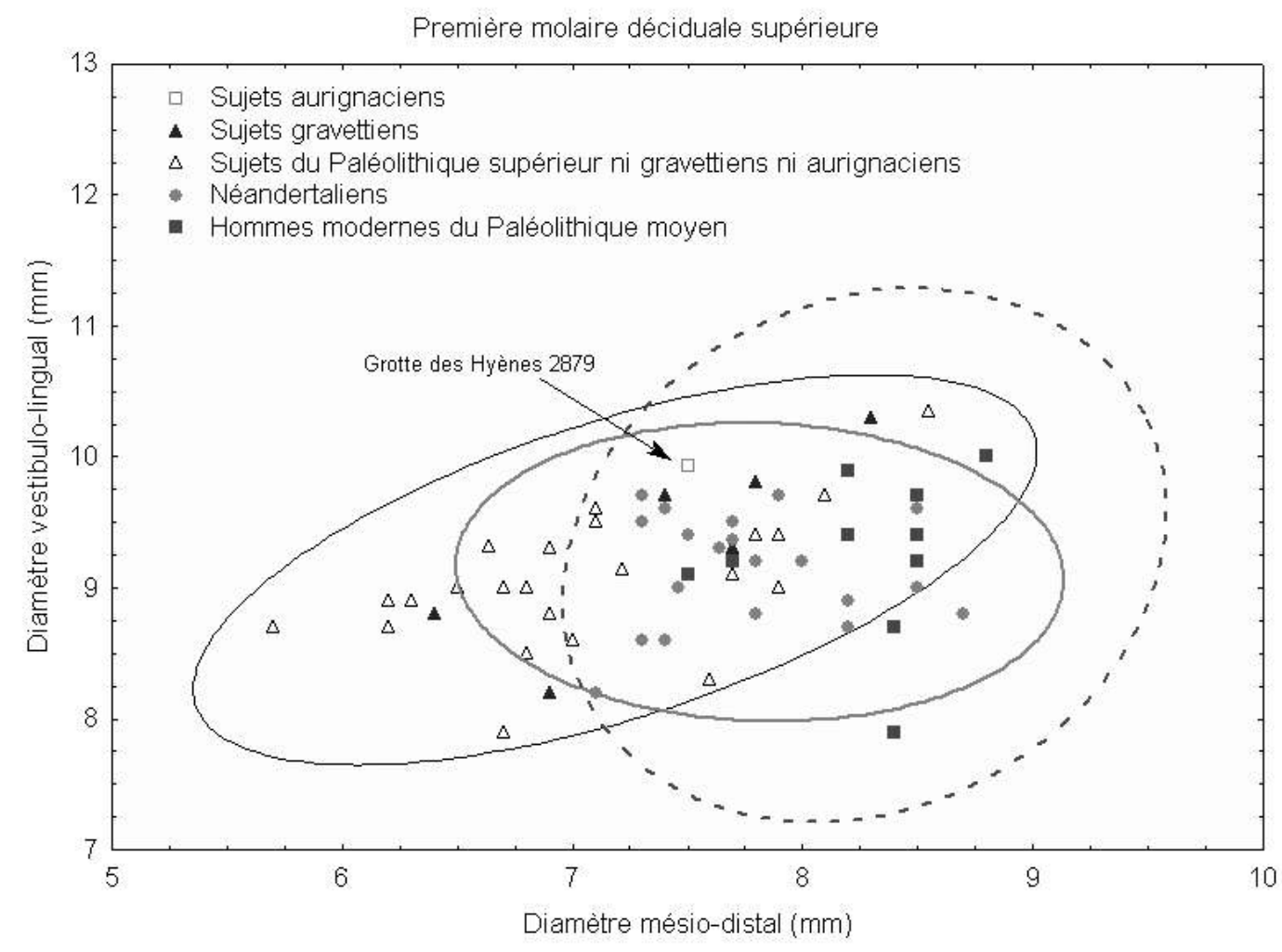

Fig. 24 - DM1 supérieure : ellipses équiprobables à $95 \%$ des échantillons de comparaison et position des Aurignaciens.

Fig. 24-UpperDM1: bivariate scatter plots. 
(forme plus carrée de la couronne). C'est particulièrement net par comparaison avec le groupe d'hommes d'anatomie moderne du Paléolithique moyen, le diamètre MD de la DM2 supérieure de Brassempouy est situé sous la limite inférieure de la variabilité du groupe.

Les VCRA indiquent que la dent 3625 est plus proche des dents gravettiennes que des DM2 supérieures du Paléolithique moyen, l'écart avec les hommes d'anatomie moderne étant le plus grand.

La zone de chevauchement entre les ellipses équiprobables des groupes fossiles est très importante (fig. 25). La dent 3625 s'inscrit dans cette zone parmi les dents dont les diamètres BL et MD sont faibles. La DM2 supérieure aurignacienne d'Hayonim se place dans la variabilité des hommes d'anatomie moderne du Paléolithique moyen avec un diamètre $\mathrm{BL}$ très élevé. La dispersion des hommes modernes du Gravettien reste notable. Les individus du Paléolithique supérieur récent semblent plus regroupés.

- La première molaire déciduale inférieure (112) a, comme les autres DM1 aurignaciennes, un diamètre BL inférieur au diamètre MD. La couronne est allongée selon l'axe MD.

Comparée aux DM1 inférieures des groupes fossiles, elle est cependant proportionnellement plus large.

Les VCRA indiquent que la dent de Brassempouy est plus proche des dents du Paléolithique moyen (en particulier de celles des Néandertaliens) que de celles des Gravettiens.

- La seconde molaire déciduale inférieure (69) a un diamètre BL inférieur au diamètre MD. La couronne est donc allongée selon l'axe MD comme celle des autres DM2 inférieures aurignaciennes. Elle se situe parmi les dents aurignaciennes les plus robustes sans atteindre cependant la robustesse de la DM2 inférieure des Rois (R50-33, ROB 115). La comparaison avec les DM2 inférieures des groupes fossiles montre que la couronne de la dent 69 est plus proche des groupes d'hommes fossiles d'anatomie moderne (Gravettiens et Qafzeh-Skhul) que des Néandertaliens. L'écart le plus faible est avec le groupe d'anatomie moderne du Paléolithique moyen.

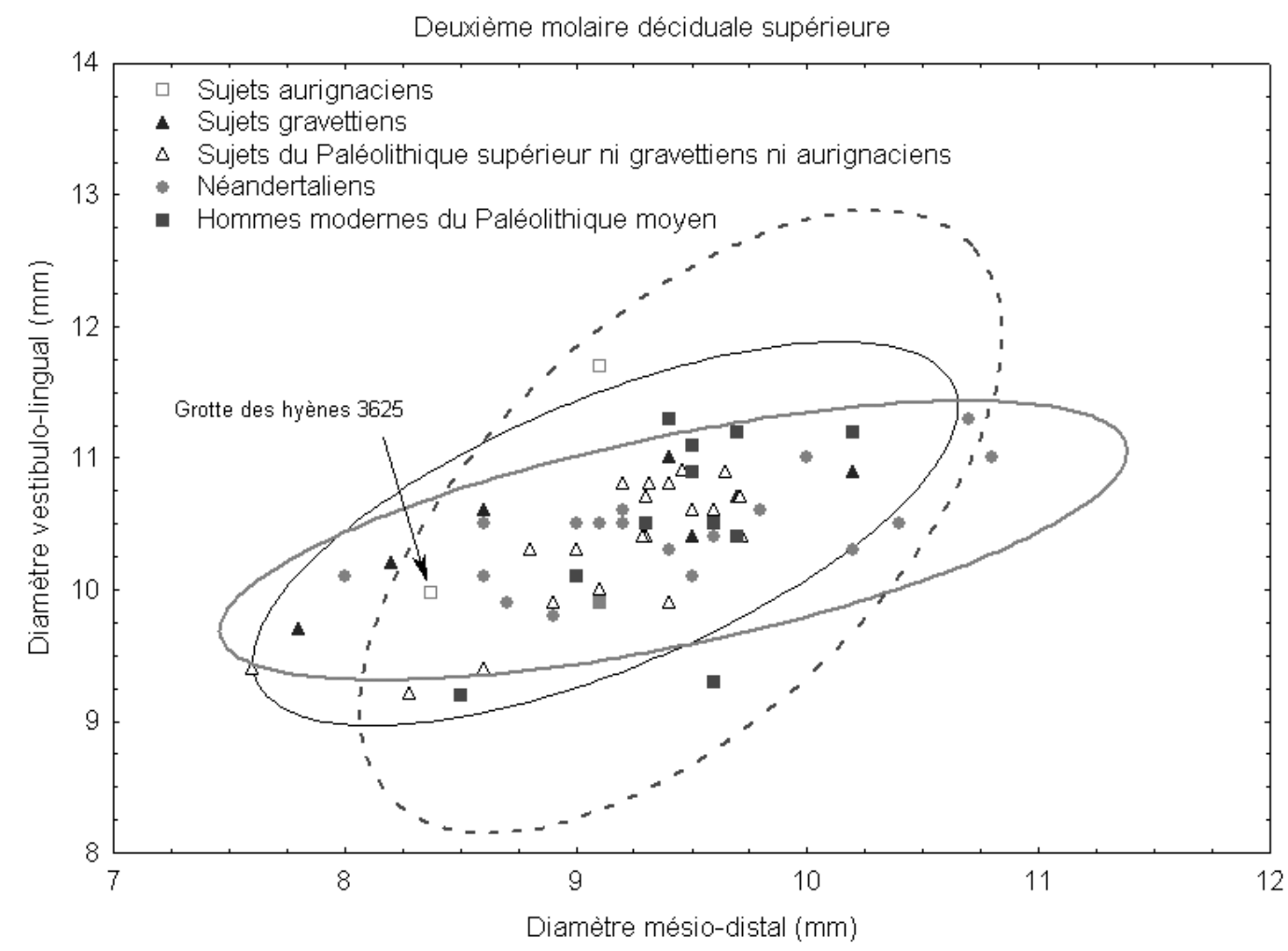

Fig. 25 - DM2 supérieure : ellipses équiprobables à $95 \%$ des échantillons de comparaison et position des Aurignaciens.

Fig. 25-Upper DM2: bivariate scatter plots 


\section{ANALYSE COMPARATIVE DES VESTIGES OSSEUX}

- Les phalanges distales des doigts II/III/IV/V?

L'impossibilité de déterminer le rayon des phalanges 302 et 344 limite l'étude comparative.

Comparées à l'échantillon d'hommes actuels (tabl. VIII), on constate que les phalanges de Brassempouy s'intègrent dans la variabilité métrique de la série d'hommes actuels de référence, quel que soit le rayon qu'on leur attribue. Les largeurs proximale et distale des deux phalanges de Brassempouy sont inférieures à la valeur moyenne de cette série. Elles sont aussi moins larges par rapport à la longueur articulaire. Elles apparaissent assez graciles. Le développement de la tubérosité de ces deux phalanges est comparable à celui observé le plus fréquemment dans les populations d'anatomie moderne.

Il existe peu de références pour le Paléolithique supérieur et le tableau VIII montre que la variabilité individuelle est forte. Ainsi le sujet de Barma Grande (Gravettien italien) a des phalanges distales plus longues, dont la diaphyse est aussi plus large au milieu et distalement que celles de Brassempouy et de Dolní Věstonice 3, 13 et 15 (Gravettien morave). Dolní Věstonice 16 occupe une position intermédiaire pour ces paramètres.

Là encore, quel que soit le rayon auquel appartiennent les deux phalanges de Brassempouy, elles sont proches de celles du Gravettien.

La plupart des auteurs (Musgrave 1973 ; Trinkaus 1983 ; Villemeur 1994 ; Smith 2000) s'accordent sur l'existence de différences significatives entre les phalanges distales néandertaliennes et celles des hommes d'anatomie moderne fossiles et actuels. Les Néandertaliens se distingueraient par :

- une longueur beaucoup plus importante de la diaphyse ;

- une grande largeur de la diaphyse au milieu et aux extrémités ;

- une hauteur de l'extrémité distale de la diaphyse proportionnellement plus importante.

La hauteur au milieu de la diaphyse par rapport à la longueur articulaire de la diaphyse serait en revanche plus faible.

Il existe bien sûr des variations individuelles (tabl. VIII).

Un travail récent (Crèvecoeur 2002) suggère que la largeur au milieu de la diaphyse et la largeur distale seraient en fait les paramètres les plus discriminants. Ces paramètres, pour les phalanges de Brassempouy, ont des valeurs très inférieures à celles des phalanges des Néandertaliens, quel que soit le rayon. Du point de vue morphologique, les deux phalanges de Brassempouy comme celles des hommes modernes gravettiens ne présentent pas la forme très élargie « en demi-cercle » de l'extrémité distale décrite chez les Néandertaliens. Elles ont plus d'affinités avec les phalanges d'hommes anatomiquement moderne qu'avec celles des Néandertaliens.

\section{- Le fragment de crâne}

Sa petitesse ne permet pas de discuter de ses affinités, les caractères pertinents n'étant pas visibles sur la portion représentée.

\section{CONCLUSIONS}

L'analyse des vestiges humains de Brassempouy apporte plusieurs types d'informations sur les Aurignaciens qui ont occupé les cavités de Brassempouy.

- Les occupations aurignaciennes de Brassempouy correspondent aux passages répétés de groupes humains sur le site (Henry-Gambier, Bon, à paraître). Les vestiges humains proviennent de plusieurs niveaux et représentent au moins quatre adultes, un adolescent, cinq immatures. Il est évident que ces chiffres ne rendent pas compte de la composition réelle des groupes qui ont fréquenté à plusieurs reprises le site entre 34000 ans BP, époque des premières occupations et 30000 ans $\mathrm{BP}$, moment où la grotte des Hyènes et la galerie Dubalen) complètement comblées ne sont plus accessibles aux hommes. On peut toutefois remarquer la présence d'immatures dont la proportion est importante.

- Des pathologies mineures, décrites dans toutes les populations du Paléolithique moyen et supérieur, ont été observées sur les dents des adultes. Il s'agit de signes de parodonthopathie tels que l'hypercémentose et le dépôt de tartre. L'hypercémentose est connue chez les Néandertaliens (Tillier et al. 1989 ; Garralda, Vandermeersch 2000) ainsi que chez les hommes du Paléolithique supérieur et en particulier sur certaines des dents aurignaciennes du site des Rois en Charente. L'hypercémentose serait chez l'homme actuel le résultat d'une réaction sur les dents soumises à des contraintes fonctionnelles trop importantes. Deux adultes présentent une anomalie superficielle et très localisée de l'amélogénèse. L'un des enfants pourrait avoir eu une 


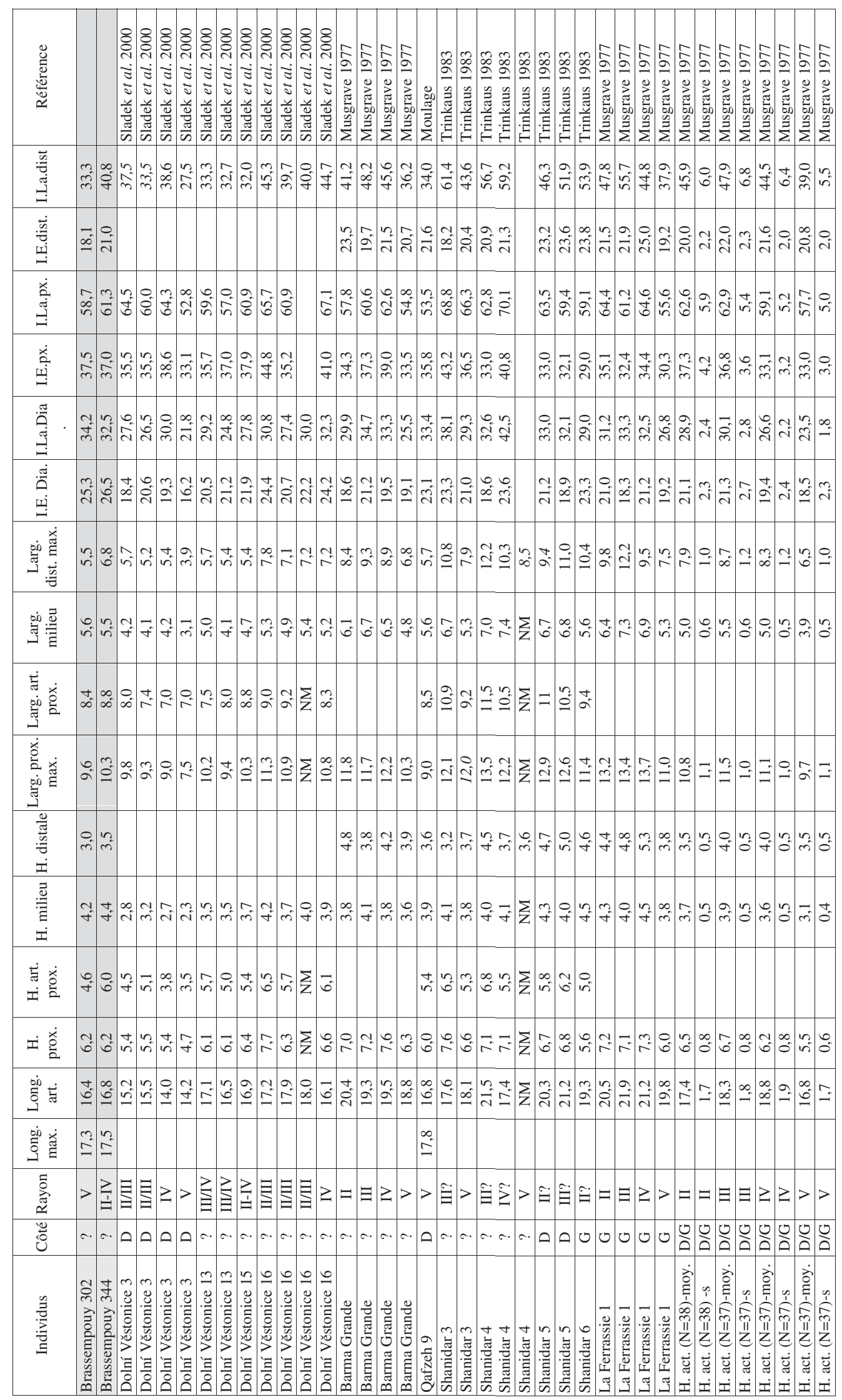

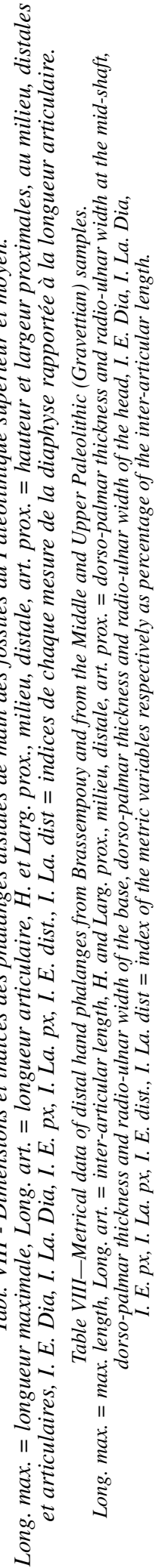


carie. Si les caries sont extrêmement rares dans les populations paléolithiques, les anomalies de l'amélogénèse, qui pourraient être liées à un trouble général d'ordre nutritionnel, sont assez fréquentes au Paléolithique supérieur (Henry-Gambier 2001).

- Les caractéristiques des fractures du fragment de crâne (316) font soupçonner une fracturation d'origine anthropique sur os frais et quatre des dents d'adultes présentent des stries de grattage sur la couronne et la racine (White, Henry-Gambier, sous presse). Trois d'entre elles ont une racine perforée, une présente une racine rainurée. Il y a donc eu prélèvement, transformation en pendeloques et conservation de certains vestiges humains. Même si ces dents ont été, comme les objets de parure (dents de carnivores, d'herbivores ou perles), abandonnées ou perdues, elles témoignent de la valeur symbolique attribuée à certains éléments squelettiques voire aux restes de certains individus. Deux autres exemples de dents humaines à racine perforée (La Combe en Dordogne et Isturitz dans les Pyrénées-Atlantiques) sont connus pour l'Aurignacien (White et al. 2003). Vlček (1992) figure une incisive a racine perforée (DV8) découverte par Absolon sur le site gravettien de Dolní Věstonice (Rép. Tchèque). Enfin, une prémolaire à racine perforée a été trouvée à Saint-Germain-la-Rivière (France) dans un niveau magdalénien daté d'environ 15000 ans BP et une molaire à racine rainurée provient du site gravettien des Vachons (Le Mort 1981 ; White et al. 2003). Aucune perforation intentionnelle de racine de dent n'a été signalée chez les hommes d'anatomie moderne du Paléolithique moyen ou chez les Néandertaliens. Chez ces derniers, une intervention sur le cadavre a cependant été mise en évidence dans plusieurs cas (Le Mort 1989 ; Garralda, Vandermeersch 2000).

Le cadre et la signification des modifications des dents ou des interventions sur le cadavre, datées du Paléolithique sont très hypothétiques (Villa 1992 ; Gambier 1996, 2000 ; Gambier, Le Mort 1996 ; White, Henry-Gambier, sous presse). Les données archéologiques sont insuffisantes pour déterminer avec certitude quels comportements sont à l'origine de ces pratiques. En particulier nous ne maîtrisons pas la part prise par les différents facteurs de destruction naturelle et/ou anthropique dans la composition des assemblages de vestiges humains paléolithiques. L'étude des sociétés historiques et actuelles révèle une très grande diversité des comportements en matière de traitement du corps et du cadavre, de pratiques funéraires et de croyances supportant ces pratiques (Thomas 1975, 1980). À titre d'hypothèse on peut avancer que les dents humaines percées relèvent de pratiques funéraires alternatives à l'inhumation primaire (Henry-Gambier 2002, White et al. 2003 ; White, Henry-Gambier, sous presse). En effet en l'absence de sépultures primaires aurignaciennes, les dents percées de Brassempouy, comme celles d'Isturitz et de La Combe, constitueraient alors le seul indice univoque d'une intervention anthropique sur le corps susceptible de relever d'un geste funéraire.

En l'état actuel des données anthropologiques et archéologiques, on constate que les Aurignaciens de Brassempouy, d'Isturitz et de la Combe ont eu un comportement original différent de celui des hommes du Moustérien (Néandertaliens et hommes d'anatomie moderne) et du Gravettien qui ont, certes selon des modalités différentes, inhumé au moins une partie de leurs défunts.

- Leur affinité taxinomique reste problématique. Dans les populations actuelles la variabilité individuelle morphologique des dents est importante. Il existe aussi une variabilité inter-population qui s'exprime à travers des différences de fréquence de caractères (Scott, Turner 1997). C'est aussi le cas dans les populations fossiles dont la variabilité est à la fois sous-estimée et mal comprise en raison des effectifs trop faibles et de l'hétérogénéité des échantillons. En effet les variations individuelles, les variations diachroniques et les variations géographiques se superposent et il est impossible d'évaluer la part de ces diverses catégories.

En ce qui concerne les dents, les Néandertaliens n'ont pas de trait morphologique spécifique. Tous les traits qui leurs sont attribués existent, avec des fréquences variables, sur les dents des populations d'anatomie moderne fossiles et actuelles. Les incisives supérieures permanentes « en pelle », le taurodontisme des molaires permanentes et déciduales, la complexité plus grande des surfaces occlusales des prémolaires ou des molaires permanentes relèvent de cette catégorie de caractères. Quant à la morphologie des molaires déciduales, elle apparaît encore moins discriminante. La spécificité néandertalienne réside dans la fréquence plus élevée de certains caractères et dans leur combinaison sur une même denture.

Il est donc impossible de déterminer les affinités d'un groupe humain à partir de dents isolées. La forme « en pelle » des incisives, considérée comme un des caractères les plus discriminants entre Néandertaliens et hommes d'anatomie moderne, illustre bien ce propos. D'une part il ne s'agit pas d'un caractère discontinu, 
d'autre part il n'est pas présent chez tous les Néandertaliens. Il peut d'ailleurs s'exprimer de manière différente sur la denture d'un même individu. C'est le cas du Moustier 1 dont les incisives centrales ne sont pas « en pelle » alors que les latérales le sont ! De la même façon, la présence d'une incisive « en pelle » parmi les incisives aurignaciennes du site des Rois ne permet pas de faire du ou des sujets des Rois, des Néandertaliens ou de leur supposer des affinités néandertaliennes !

Pour ces raisons, l'absence d'incisive en «pelle » à Brassempouy ne constitue pas une preuve univoque en faveur d'une attribution de ces dents aux hommes d'anatomie moderne.

Par leur morphologie, les dents isolées de Brassempouy et celles des autres Aurignaciens s'intègrent aussi bien dans la variabilité des populations d'anatomie moderne du Paléolithique moyen et supérieur que dans celle des populations d'anatomie moderne actuelles. Cette conclusion vaut aussi, à notre avis, pour toutes les autres dents isolées aurignaciennes.

Les données métriques ne permettent pas non plus de trancher.

La seule différenciation nette est celle qui existe entre les dents de Brassempouy et celles des deux populations actuelles de référence. Les dents déciduales et permanentes de Brassempouy sont plus volumineuses et plus robustes que celles des groupes d'hommes actuels que nous avons utilisés. Ce résultat illustre la tendance générale à la réduction de la denture dans la lignée humaine.

Les dents de Brassempouy sont très proches des autres dents aurignaciennes. On peut toutefois remarquer que, parmi les dents aurignaciennes (y compris celles de Brassempouy), certaines des dents des Rois ont une couronne particulièrement robuste. En revanche, la seconde molaire inférieure de Brassempouy, comme celle des autres Aurignaciens, n'atteint pas les dimensions de la seconde molaire permanente inférieure d'Oase 1 décrite par Trinkaus et al. (2003).

Pour chacun des paramètres métriques, la majorité des dents de Brassempouy est située entre les limites de $95 \%$ de la variabilité des groupes fossiles de référence.

Seules quatre dents $(262,3040,3625,2879)$ ont au plus deux paramètres en dehors de la variabilité d'un groupe fossile.

- L'incisive latérale 262 sort de la variabilité néandertalienne par la valeur de l'indice de la couronne en raison d'un diamètre BL faible. Selon P. Semal (1988), le développement bucco-lingual des incisives serait un caractère dérivé néandertalien. $\mathrm{Si}$ on accepte cette conclusion, la dent 262 n'appartiendrait pas à la population néandertalienne. Pour l'ensemble des paramètres, cette incisive latérale est en outre plus proche de la distribution de la population de Qafzeh-Skhul estimée à partir de l'échantillon.

- La P2 inférieure 3040 de Brassempouy est exclue de la variabilité des hommes d'anatomie moderne du Paléolithique moyen par la valeur de l'indice de la couronne. Elle serait plus proche pour l'ensemble des paramètres de la distribution de la population néandertalienne estimée à partir de l'échantillon.

- La DM1 supérieure 2879 est exclue de la variabilité des hommes d'anatomie moderne de Qafzeh. Elle est plus proche, pour les quatre paramètres, de la distribution de la population d'hommes d'anatomie moderne du Gravettien estimée à partir de l'échantillon.

- La DM2 supérieure 3625 sort de la variabilité des hommes d'anatomie moderne de Qafzeh. Elle est plus proche, pour l'ensemble des paramètres, de la distribution de la population d'hommes d'anatomie moderne du Gravettien estimée à partir de l'échantillon.

La position des autres dents (quel que soit le paramètre de référence) par rapport à la distribution de chaque population fossile estimée à partir de l'échantillon est variable. L'incisive centrale supérieure 1046, la canine supérieure 441, la seconde molaire supérieure 542, la première molaire déciduale inférieure 112 sont plus proches de la distribution de la population néandertalienne. La seconde molaire permanente supérieure 884 est plus proche de la distribution de la population d'anatomie moderne gravettienne. La seconde molaire déciduale inférieure 69 est plus proche de la distribution de la population de Qafzeh.

Ces résultats, en apparence paradoxaux, illustrent la difficulté d'une diagnose taxinomique à partir de dents isolées dès lors qu'il existe un recouvrement important entre les variabilités des dents des groupes fossiles de référence.

Les vestiges humains de Brassempouy n'apportent donc pas d'éclaircissement sur l'identité des populations de l'Aurignacien ancien du Sud-Ouest de la France.

\section{Remerciements}

Les fouilles de Brassempouy se déroulent depuis 1981 grâce à l'aide financière du SRA d'Aquitaine, du Ministère de la Culture, du Conseil général des Landes, du 
SIVOM d'Amou, de la commune de Brassempouy et de la Société des Amis de Brassempouy.

Nous remercions tous ceux qui, à un titre ou à un autre, ont permis la réalisation de ce travail : H. Delporte, directeur des fouilles de Brassempouy de 1981 à 1994, D. Buisson, directeur des fouilles de Brassempouy de 1995 à 1996, pour leur soutien ; J.-J. Cleyet-Merle et P. Perin, directeurs du MNP (Les-Eyzies-de-Tayac) et du MAN (Saint-Germain-en-Laye) qui nous ont accueilli à plusieurs reprises pour étudier du matériel ; les membres de l'équipe de recherche du site ainsi que les fouilleurs bénévoles, étudiants ou amateurs, qui ont permis la découverte de ces vestiges, P. Semal qui nous a autorisé à utiliser ces données et M. Seurin (IE CNRS) qui a réalisé la carte.

Enfin ces recherches se sont en partie déroulées grâce au soutien financier du CNRS dans le cadre du programme OHLL et de la European Science Foundation dans le cadre du programme OMLL. 


\section{BIBLIOGRAPHIE}

BAILEY (S.E.) 2002, Neanderthal Dental Morphology: Implications for modern Human origins, $\mathrm{PhD}$, Arizona State University, $239 \mathrm{p}$.

BAy (R.), CADENAT (P.) 1973, Une molaire humaine à Brassempouy (Landes), Paris, L'Anthropologie 77, 3-4 : 345-352.

Bermudez de Castro (J.L.), Rosas (A.), Nicolas (M.E.) 1999, Dental remains from Atapuerca-TD6 (Gran Dolina site, Burgos, Spain), Journal of Human Evolution 37: 523-566.

Bon (F.) 2002, L'Aurignacien entre Mer et Océan, Réflexion sur l'unité des phases anciennes de l'Aurignacien dans le sud de la France, Mémoire de la Société préhistorique française 29, $253 \mathrm{p}$.

Bon (F.) Gambier (D.), Ferrier (C.), Gardère (P.) 1998, Gisement de Brassempouy (Landes) : les recherches de 1995 à 1997, bilan et perspectives, Bulletin de la Société de Borda 449 : 203-222.

Bordes (J.-G.) 2002, Les interstratifications Châtelperronien/ Aurignacien du Roc de Combe et du Piage (Lot, France), Analyse taphonomique des industries lithiques, Implications Archéologiques, Thèse 2614, Université Bordeaux 1, 382 p.

Boulestin (B.) 1998, Approche taphonomique des restes humains, Le cas des Mésolithiques de la grotte des Perrats (Agris), Charente, Thèse 2004, Université Bordeaux 1, 2 vol, $448 \mathrm{p}$.

Brabant (H.) Salhy (A.) 1962, La paléostomatologie en Belgique et en France, Acta Stomatologica 59 : 285-355.

Buisson (D.) 1996, Brassempouy : présentation du site et problèmes posés par les fouilles récentes, in Pyrénées Préhistoriques : Arts et Sociétés, CTHS, Paris, p. 423-437.

Buisson (D.), Delporte (H.) 1990, Existence du Castelperronien à Brassempouy (Landes), in C. Farizy (dir.), Paléolithique moyen récent et Paléolithique supérieur ancien en Europe, Ruptures et transitions : examen critique des documents archéologiques, Mémoires du Musée de Préhistoire d'Ile-de-France 3, APRAIF, Nemours, p. 189-193.

Champagne (F.), Espitalí́ (R.) 1981, Le Piage, site préhistorique du Lot, Mémoire de la Société Préhistorique française 15, $205 \mathrm{p}$.
Churchill (S.E.), SMith (H.F.) 2000, Makers of the Early Aurignacian of Europe, Yearbook of Physical Anthropology 43: 61-115.

Cleuvenot (E.), HouËt (F.) 1993, Proposition de nouvelles équations d'estimation de stature applicables pour un sexe indéterminé, et basées sur les échantillons de Trotter et Gleser, Bulletins et Mémoires de la Société d'Anthropologie de Paris, n.s., 5, 1-2 : 245-255.

CRÈVECEUR (I.) 2002, Étude morphologique et biométrique des ossements de mains retrouvés dans la couche castelperronienne du gisement de Saint-Césaire (CharenteMaritime), Mémoire DEA, Université Bordeaux 1, Laboratoire Anthropologie des Populations du Passé, 70 p.

DelPorte (H.) 1967, Brassempouy : ses industries d'après la collection Piette (Musée des Antiquités nationales), Zephyrus (Universidad de Salamanca) XVIII : 5-41.

Delporte (H.) 1980, Brassempouy, La grotte du Pape, station préhistorique, Il y a 20000 ans... L'Art, Association culturelle de Contis (Landes), $75 \mathrm{p}$.

Delporte (H.) 1987, E. Piette, Histoire de l'art primitif. Précédé de Piette pionnier de la préhistoire, Picard, Paris, $275 \mathrm{p}$.

DelPoRte (H.) 1996, Brassempouy : Histoire d'un gisement, in Pyrénées Préhistoriques : Arts et Sociétés, CTHS, Paris, p. $415-421$.

Dubalen (P.E.) 1881, Les abris sous roche de Brassempouy (Chalosse, Landes), Matériaux pour l'Histoire Primitive et Naturelle de l'Homme 16 : 284.

Gambier (D.) 1992, Vestiges humains du Paléolithique supérieur : inventaire et description préliminaire des spécimens inédits des collections du Musée national de Préhistoire, Les Eyzies-de-Tayac, Paléo 4 : 91-100.

GAMBIER (D.) 1996, Les pratiques funéraires au Magdalénien dans les Pyrénées françaises, in Pyrénées préhistoriques : Art et Société, CTHS, Paris, p. 263-277.

GAMBIER (D.) 1997, Modern Humans at the beginning of the Upper Palaeolithic in France: The range of anthropological data, in G.A. Clark, C.M. Willermet (eds), Conceptual Issues in Modern Human Origins Research, Aldine de Gruyter, New York, p. 117-131. 
GAMBIER (D.) 2000, Aurignacian children and mortuary practice in Western Europe, Anthropologie (Brno) 38, 1: 5-21.

Gambier (D.), HouËt (F.), Tillier (A.) 1990, Dents de Font de Gaume (Châtelperronien et Aurignacien) et de La Ferrassie (Aurignacien ancien), Paléo 2 : 143-152.

Gambier (D.), Le Mort (F.) 1996, Modifications artificielles et série anciennes : possibilités et limites de l'interprétation palethnologique, Bulletins et Mémoires de la Société d'Anthropologie de Paris, n.s., 8, 3-4 : 245-260.

GAMBIER (D.), SACCHI (D.) 1991, Sur quelques restes humains leptolithiques de la grotte de la Crouzade, Aude, L'Anthropologie 95, 1: 155-179.

Gambier (D.), Tisnerat (N.), Valladas (H.) 2000, Datations de vestiges humains présumés du Paléolithique supérieur par la méthode du Carbone 14 en spectrométrie de masse par accélérateur, Paléo 16 : 1-11.

Garralda (M.D.), Tillier (A.-M.), Vandermeersch (B.), Cabrera (V.), Gambier (D.) 1992, Restes humains de La Cueva de El Castillo (Santander, Espagne), Anthropologie (Brno) 30-2 : 159-164.

Garralda (M.D.), VANDERMEersch (B.) 2000, Les Néanderthaliens de la grotte de Combe-Grenal (Domme, Dordogne, France), Paléo 12 : 213-260.

Glen (E.), KacZanowsKi (K.) 1982, Human remains, in J.K. Koslowski (ed.), Excavation in the Bacho Kiro Cave (Bulgaria): final report, Pantstwowe Wydawnictwo Naukowe, Warsaw, p. 75-79.

HeIM (J.-L.) 1976, Les hommes fossiles de La Ferrassie, Archives de l'Institut de Paléontologie humaine 35, Masson, Paris, $331 \mathrm{p}$.

Henri-Martin 1923, L'homme fossile de La Quina, Archives de morphologie générale et expérimentale, Doin, Paris, $263 \mathrm{p}$.

HENRY-GAMBIER (D.) 2001, Les enfants de Grimaldi (grotte des Enfants, site des Baoussé-Roussé, Italie), Anthropologie et palethnologie funéraire, CTHS/RMN, Paris, (D. HenryGambier, M.-A. Courty, É. Crubézy, B. Kervazo), 177 p.

Henry-Gambier (D.) 2002, Les fossiles de Cro-Magnon (Les- Eyzies-de-Tayac, Dordogne) : nouvelles données sur leur position chronologique et leur attribution culturelle, Bulletins et Mémoires de la Société d'Anthropologie de Paris, n.s., 14, 1-2 : 89-112.
Henry-GAMBIER (D.), Bon (F.), (à paraître), L'Aurignacien ancien de la grotte des Hyènes (Brassempouy, Landes), Sous la direction de D. Henry-Gambier et F. Bon (avec la collaboration de C. Ferrier, M. Fontugne, P. Gardère, H. Katz, C. Le Tourneux, R. Mensan, Y. Potin, R. White).

KIESER (J.A.) 1990, Human adult odontometrics, Cambridge University Press, Cambridge, 194 p.

LAPORTERIE (J.) DE 1892, La grotte du Pape à Brassempouy, Association Française pour l'Avancement des Sciences, $21^{\mathrm{e}}$ session, Pau, $1: 25-261$.

Le Mort (F.) 1981, Dégradations artificielles sur des os humains du Paléolithique, Thèse de 3 c cycle, Université Pierre et Marie Curie, Paris, 204 p.

Le Mort (F.) 1989, Traces de décharnement sur les ossements néandertaliens de Combe-Grenal (Dordogne), Bulletin de la Société Préhistorique Française 86, 3 : 79-87.

Maureille (B.), Rougier (H.), HouËt (F.), VANDERMEersch (B.) 2001, Les dents inférieures du Néandertalien Regourdou 1 (commune de Montignac, Dordogne) : analyses métriques et comparatives, Paléo 13 : 183-200.

Merlet (J.-C.) 1990, Brassempouy : la collection De Laporterie au musée de Dax, Bulletin de la Société Préhistorique Française 87, 7 : 201-205.

Molleson (T.), Cox (M.) 1993, The Spitalfields project, vol. 2: The Anthropology, The Middling Sort, CBA Research Report 86, New York, Council for British Archaeology, $231 \mathrm{p}$.

MusgraVe (J.H.) 1973, The phalanges of Neanderthal and Upper Palaeolithic hands, in M. Day (ed.), Human Evolution, Taylor and Francis LTD, London, p. 59-85.

MusgraVe (J.H.) 1977, The Neandertals from Krapina, northern Yugoslavia: An inventory of the hand bones, Zeitschrift für Morphologie und Anthropologie 68, 2: $150-171$

PatTe (E.) 1959, La dentition des Néanderthaliens, Annales de Paléontologie 45, 159 p.

PietTe (E.) 1895, La station de Brassempouy et les statuettes humaines de la période glyptique, L'Anthropologie $6: 129-151$.

PIETTE (E.) 1896, Fouilles faites à Brassempouy en 1895, Bulletins et Mémoires de la Société d'Anthropologie de Paris 7 : 659-663. 
PietTe (E.), DE LAPORTERIE (J.) 1895, Les fouilles de Brassempouy en 1894, Bulletins et Mémoires de la Société d'Anthropologie de Paris 5 : 633-648.

PietTe (E.), DE LAPORTERIE (J.) 1897, Études d'ethnographie préhistorique, IV, Fouilles à Brassempouy en 1896, L'Anthropologie 8 : 165-176.

Piette (E.), DE LAPORTERIE (J.) 1898, Étude d'ethnographie préhistorique, V, Fouilles à Brassempouy en 1897, L'Anthropologie 9, 5 : 531-556.

ScotT (G.R.), TuRner (C.G.) 1997, The Anthropology of Modern Human Teeth, Dental Morphology and its Variation in Recent Human Populations, Cambridge University Press, Cambridge, $382 \mathrm{p}$.

Semal (P.) 1988, Évolution et variabilité des dimensions dentaires chez Homo sapiens neanderthalensis, Ed. CEDARC, Vinoinval, $112 \mathrm{p}$.

Sladek (V.), Trinkaus (E.), Hillson (S.W.), Holliday (T.W.) 2000, The people of the Pavlovian, The Dolní Věstonice Studies 5, Ed. Institute of Archaeology, Academy of Sciences of the Czech Republic, Brno, 244 p.

SMith (F.H.) 1976, The Neandertal remains from Krapina, A descriptive and comparative study, University of Tennessee, Report of Investigations 15, Knoxville, 359 p.

SMITH (S.L.) 2000, Shape variation of the human pollical distal phalanx and metacarpal, American Journal of Physical Anthropology 113: 329-348.

Svoboda (J.A.), Van Der Plicht (J.), Kuzela (V.) 2002, Upper Palaeolithic and Mesolithic human fossils from Moravia and Bohemia (Czech Republic): some new C14 dates, Antiquity 76: 9577-9962.

Thomas (L.V.) 1975, Anthropologie de la mort, Payot, Paris, $538 \mathrm{p}$.

Thomas (L.V.) 1980, Le cadavre, De la biologie à l'anthropologie, Complexe, Bruxelles, 214 p.

Tillier (A.-M.) 1979, La dentition de l'enfant moustérien Châteauneuf 2 découverte à l'abri de Hauteroche, L'Anthropologie 83 : 417-438.

Tillier (A.-M.) 1983, L'enfant néanderthalien du Roc de Marsal (Campagne du Bugue, Dordogne) : le squelette facial, Annales de Paléontologie 69, 2 : 725-727.
TILlier (A.-M.) 1999, Les enfants moustériens de Qafzeh, interprétation phylogénétique et paléoauxologique, Cahiers de Paléoanthropologie, CNRS, Paris, 239 p.

Tillier (A.-M.), Arensburg (B.), Duday (H.) 1989, La mandibule et les dents du Néanderthalien de Kébara (Homo 2), Mont Carmel, Israël, Paléorient 15 : 39-58.

TILlier (A.-M.), TiXIER (J.) 1991, Une molaire d'enfant aurignacien à Ksar Aqil (Liban), Paléorient 17, 1 : 89-93.

TrinKaus (E.) 1983, The Shanidar Neanderthal, Academic Press, New York, 502 p.

Trinkaus (E.), Moldovan (O.), Milotas (S.), Bilgar (A.), Sarcina (L.), Athreya (S.), Bailey (S.E.), Rodrigo (R.), Mircea (G.), Higham (T.), Ramsey (C.B.), Van der Plicht (J.) 2003, An Early Modern Human from Pestera cu Oase, Romania, Proceedings of the National Academy of Sciences 100, 20: 11231-11236.

Twiesselmann (F.), Brabant (H.) 1967, Observations sur les dents et les maxillaires d'une population ancienne d'âge franc de Coxyde, Bulletin du Groupement International de Recherches en Sciences Stomatologiques 3-4 : 1-139.

UbelaKer (D.H.) 1978, Human skeletal Remains, Excavation analysis, interpretation, Washington, Taraxacum, $178 \mathrm{p}$.

VALLOIS (H.V.) 1958, Les restes humains d'âge aurignacien de la grotte des Rois, Charente, Bulletins et Mémoires de la Société d'Anthropologie 10e série, 9 : 139-159.

VANDERMEERSCH (B.) 1981, Les hommes fossiles de Qafzeh (Israël), Cahiers de Paléoanthropologie, CNRS, Paris, $319 \mathrm{p}$.

VILla (P.) 1992, Cannibalism in Prehistoric Europe, Evolutionary Anthropology 1: 93-104.

VILLEMEUR (I.) 1994, La main des Néanderthaliens. Comparaison avec la main des hommes de type moderne, Morphologie et mécanique, Cahiers de Paléoanthropologie, CNRS, Paris.

VLčEK (E.) 1992, Lovci mamutůz Dolnich Věstonic, Acta Musei National Pragae, B XLVIII, 1- 4: 7.

White (R.), Henry-Gambier (D.), sous presse, Modifications artificielles des vestiges humains de l'Aurignacien ancien de la grotte des Hyènes (Brassempouy, Landes). Quelle signification ? Actes du colloque international «En el centenario de la cueva de El Castillo : el ocaso de los Neandertales » Santona, 18-20 septembre 2003, 
Universidad Nacional de Educacion a Distancia, Centro asociado de Cantabria.

White (R.), Normand (C.), Henry-Gambier (D.) 2003, Human-tooth ornaments from the French early Aurignacian: implications for early Upper Paleolithic treatment of the dead, Paleo anthropology Society (Abstract), www. Paleoanthro. org.

WolPOFF (M.H.) 1979, The Krapina dental remains, American Journal of Physical Anthropology 50: 67-114.
WolPOFF (M.H.), SMith (F.H.), MALEZ (M.), RADOVCIC (J.), RukAVINA (D.) 1981, Upper Pleistocene hominid remains from Vindija Cave, Croatia, Yugoslavia, American Journal of Physical Anthropology 54: 499-545.

ZilhaO (J.), D'ERrico (F.) 1999, The chronology and taphonomy of the earliest Aurignacian and its implications for the understanding of Neandertal extinction, Journal of World Prehistory 13: 1-68. 


\section{Composition des échantillons gravettiens et moustériens de comparaison (par dent) \\ Incisive centrale supérieure}

Homo sapiens sapiens du Gravettien : Arene Candide I, Dolní Věstonice 13, 14, 15, Lagar Velho 1, Paglicci (21 d) 15, Pataud 1, Předmostí 1, 2, 3, 4, 5, 9, 10, 14.

Néandertaliens : Amud 01, Angles-sur-l’Anglin, Arcy NN5 (A9), Carihuela (Pinar7), Châteauneuf 2, Combe Grenal 05, 11, 27, Cotencher, Cova Negra 7856, Devil's Tower- Gibraltar 2, Genay 1, Hortus 7, 8, 9, 10, La Ferrassie 2, La Quina H18, H 5, Le Moustier 1, Monsempron 4, Saint-Brais II, Saint-Césaire 1, Shanidar 1, 2, Subalyuk 2, Vindija 290.

Homo sapiens sapiens du Paléolithique moyen : Qafzeh 4, 5, 6, 7, 9, 10, 11, 12, 15, 21, Skhul 1, 4, 5, 7.

\section{Incisive latérale supérieure}

Homo sapiens sapiens du Gravettien : Arene Candide I, Dolní Věstonice 14, 15, Lagar Velho 1, Paglicci (21 d) 15, Pataud 1, Pavlov 22, Předmostí 3, 4, 5, 7, 9, 14.

Néandertaliens: Amud 01, Arcy NN6 (B9), Bau de 1'Aubesier 04, 09, Carihuela (Pinar7), Châteauneuf 2, Combe Grenal 10, Genay 01, Grotte Boccard 76 B14 C5 26, Hortus 07, 08, 10, 11, Kébara 27, La Ferrassie 02, La Quina H18, H05, Le Moustier 1, Monsempron 03, Rescoundudou V, Saint-Césaire 01, Shanidar 01, 02, Spy 2, Subalyuk 2, Vindija 289.

Homo sapiens sapiens du Paléolithique moyen : Qafzeh 05, 06, 07, 08, 09, 11, 12, 15, 21, Skhul 01, 04, 05, 07.

\section{Canine supérieure}

Homo sapiens sapiens du Gravettien : Arene Candide I, Dolní Věstonice 03, 13, 14, 15, Lagar Velho 1, Paglicci (21d) 15, Předmostí 1, 10, 14, 3, 4, 5, 7, 9.

Néandertaliens : Amud 01, 02, Cotencher, Genay 01, Hortus 03, 08, 09, Kulna, La Croze del Dua 1, La Ferrassie 02, La Quina H05, Le Moustier 1, Monsempron 03, Saint-Césaire 01, Shanidar 01, 02, 03, 06, Spy 1, Spy 2, Vindija 287.

Homo sapiens sapiens, du Paléolithique moyen : Qafzeh 05, 06, 07, 08, 09, 11, 12, 21, Skhul 01, 02, 04, 05, 06.

\section{Deuxième prémolaire inférieure}

Homo sapiens sapiens du Gravettien : Arene Candide I, Dolní Věstonice 03, 13, 14, 15, Paglicci (21 d) 15, Pavlov 01, 03, Předmostí 10, 14, 2, 4, 5, 7, 9 .

Néandertaliens : Agut 01, Amu 01, Arcy 17, Bau de l'Aubesier 01, Châteauneuf 2, Circéo IV (Grotta de Fossellone 1), Combe Grenal 04, 08, 29, El fate 02, Fosselone 03, Genay 01, Hortus 02, 05, Kébara 02, La Croze del Dua 2, La Quina H05, H09, Le Moustier 1, Le Placard 22, Le Portel ? 01? : 3, 9, 11, 7, 5, 1, 4, 8, Monsempron 01, Ochoz 1, PetitPuymoyen 1, Régourdou, Saint-Césaire 01, Shanidar 01, 02, 04, 06, Sipka, Spy 1, 2, Subalyuk 1, Vergisson NN (4), Vindija 231.

Homo sapiens sapiens du Paléolithique moyen : Qafzeh 07, 08, 09, 11, Skhul 02, 04, 05.

\section{Seconde molaire supérieure}

Homo sapiens sapiens du Gravettien : Arene Candide I, Dolní Věstonice 03, 13, 14, 15, Paglicci (21 d) 15, Pataud 1, Pavlov 01, 02, Předmostí 1, 3, 4, 5, 7, 9, 10, 14.

Néandertaliens : Amud 01, Arcy 09 - mx III, Baume de Peyrards 01, Châteauneuf 2, Cotencher, Genay 01, Grotta de li Poggi, Hortus 03, La Croze del Dua 3, La Quina H18, H05, Le Moustier 1, Les Rivaux 01, Monsempron 03, PetitPuymoyen 2, Saint-Césaire 01, Shanidar 01, 02, 06, Spy 1, 2, Vindija 229, 259.

Homo sapiens sapiens du Paléolithique moyen : Qafzeh 06, 07, 09, 10, 11, Skhul 02, 04, 05, 07, 10. 


\section{Seconde molaire inférieure}

Homo sapiens sapiens du Gravettien : Arene Candide I, Dolní Věstonice 03, 13, 14, 15, 37, Les Battuts 1, Les Marronniers 1, Les Vachons 1, Paglicci (21 d) 15, Pataud 1, Pavlov 1, Předmostİ 1, 2, 4, 7, t 9, 10, 14, 18.

Néandertaliens : Amud 01, 05, Arcy NN3 (D4), Arcy 15, Banolas, Castillo B, Châteauneuf 2, Circéo IV (Grotta de Fossellone 1), Devil's Tower-Gibraltar 2, El Fate 12, El Fate 02, Fosselone 03, Genay 01, Grotte Boccard 78 B13 C5 66, Guattari III (Monte Circeo B ou III), Hortus 04, 05, Kébara 02, La Payre 0 à 1, La Quina H05, H09, Le Moustier 1, Le Portel 32, Macassargues 02, Maglie 01 ou Fondo Cattie 01, Monte Fenera 2 (Ciota Ciara 01), Ochoz 1, Petit-Puymoyen 1, Régourdou, Rochelot 1, Saint-Césaire 01, Shanidar 01, 02, 06, Soulabé 01, Spy 1, 2, Subalyuk 1, Vindija 206, 231.

Homo sapiens sapiens du Paléolithique moyen : Qafzeh 07, 08, 09, 11, Skhul 04, 05, 07.

\section{Première molaire déciduale supérieure}

Homo sapiens sapiens du Gravettien : Dolní Věstonice 36/2, Lagar Velho, Pataud 3L, Pavlov 06/1, 11, Roc de Combe Capelle 03 .

Néandertaliens : Amud 03, Châteauneuf/Charente 2 (Haute-Roche), Devil 's Tower (Gibraltar 2), Engis 2, Kébara 01, 03, 04, 13, 25, La Ferrassie 08, La Quina H18, Le Portel 26, 31, Pech-de-l'Azé, Roc de Marsal, Shanidar 07, Subalyuk 2.

Homo sapiens sapiens du Paléolithique moyen: Qafzeh 04, 10, 12, 14, 15, 21, Skhul 01.

\section{Seconde molaire déciduale supérieure}

Homo sapiens sapiens du Gravettien : Dolní Věstonice 36/3, Labattut 3, Lagar Velho, Paglicci (21 d) 15, Pataud 3L, Pavlov 06/2, Pavlov 12, Roc de Combe Capelle 03.

Néandertaliens: Amud 03, Bau de L'Aubesier 07, Carihuela (Pinar 7), Châteauneuf/Charente 2 (Haute-Roche), Devil's Tower (Gibraltar 2), Engis 2, Grotta del Cavallo C, Hortus 3, Kébara 01, 25, La Ferrassie 08, La Quina H18, Le Portel 29, Pech-de-l'Azé, Rescoundudou 04, Roc de Marsal, Shanidar 07, Subalyuk 2.

Homo sapiens sapiens du Paléolithique moyen : Qafzeh 04, 10, 12, 14, 15, 21, Skhul 01.

\section{Première molaire déciduale inférieure}

Homo sapiens sapiens du Gravettien : Lagar Velho, Le Rouset 1, Les Marronniers 2

Néandertaliens : Châteauneuf/Charente 1, 2 (Haute-Roche), Combe Grenal 01, Devil's Tower (Gibraltar 02), Engis 2, Kébara 01, 04, La Ferrassie 08, Le Portel 19, Pech-de-l'Azé, Rescoundudou 02, Roc de Marsal, Shanidar 07, TeshikTash.

Homo sapiens sapiens du Paléolithique moyen : Qafzeh 04, 10, 12, 13, 14, 15, 21, Skhul 01.

\section{Seconde molaire déciduale inférieure}

Homo sapiens sapiens du Gravettien : Dolní Věstonice 36/6, Lagar Velho, Le Rouset 1, Pavlov 07, 08, 09, 10.

Néandertaliens: Amud 03, Châteauneuf/Charente 2 (Haute-Roche), Combe Grenal 01, Cova Negra 7755, Devil's Tower (Gibraltar 2), Engis 2, Grotta del Caballo A, Gruta das Salemas, Hortus 02, Kébara 01, 04, La Ferrassie 04bis, 08, Pech-de-l'Azé, Rescoundudou 01, Roc de Marsal, Shanidar 07, Teshik-Tash.

Homo sapiens sapiens du Paléolithique moyen : Qafzeh 04, 10, 12, 15, 21, Skhul 01, 10. 


\section{Spécimens du Paléolithique supérieur ancien et récent utilisés pour les ellipses équiprobables}

Incisive latérale supérieure: Arene Candide 01, 02, 04, 05, Badegoule -W3, -W4, Canecaude 2, Cap-Blanc, Dolní Věstonice 14, 15, Gazel 3, Isturitz 92, Lafaye 24, Lagar Velho 01, Paglicci (21 d) 15, Pataud 1, Předmostí 3, 4, 5, 7, 9, 14, Roc de Cave 1, Romanelli 16, Romito 2, 4, 6, Saint-Germain-la-Rivière 1, Vado all'Arancio 1.

Deuxième prémolaire inférieure: Arene Candide 01, 02, 04, 05, Cap-Blanc, 03, 13, 14, 15, Gazel 4, Isturitz 82, 88, 90, Lachaud 0, 1, Lafaye 24, Paglicci (21 d) 15, Pavlov 01, 03, Předmostí 2, 4, 5, 7, 9, 10, 14, Roc de Cave 1, Romito 2, Saint-Germain-la-Rivière 01, 09, Vado all'Arancio 1.

Première molaire déciduale supérieure : Arene Candide 11, 06, 08, Bois-Ragot 3, 1, Dolní Věstonice 36/2, Grotte des Enfants 1, 2, La Madeleine 04, Lafaye 25, Lagar Velho, Le Figuier, Le Morin 5, Le Placard 32, 33, Pataud 3L, Pavlov 06/1, 11, Pis de la Vache 1, Roc de Combe Capelle 3, Rochereil 1, Romanelli 10, Saint-Germain-la-Rivière 07, Vado All'Arancio 2.

Seconde molaire déciduale supérieure: Canecaude 3, Arene Candide 06, 08, Cabène 1, 2, 3, Dolní Věstonice 36/3, Fauroux 1, Grotte des Enfants 1, La Madeleine 4, Labattut 3, Lafaye 25, Lagar Velho, Le Figuier, Le Placard 32, Les Peyrugues 2, Moulin Neuf 1, Paglicci (21 d) 15, Pataud 3L, Pavlov 06/2, 12, Ranchot 2, 3, Roc de Combe Capelle 3, Rochereil 1, Saint-Germain-la-Rivière 07, Troubat 2, Vado All'Arancio 2.

Annexe II.

Annex II. 